\title{
Das Vorsorgeverhältnis
}




\title{
Soziale Sicherheit durch Vorsorge - Sicherheit als Verfassungsprinzip des Sozialstaats und das „Vorsorgeverhältnis“ als rechtliches Gehäuse ihrer Vorsorgestandards
}

\author{
Rainer Pitschas
}

A. Sicherheit als Verfassungsprinzip und soziale Vorsorge als ihr Gewährleistungsmodus

I. Der Sozialstaat als Staatsziel

II. Sicherheit als Verfassungsprinzip des Sozialstaats

1. Das Verfassungsprinzip „Sicherheit“"

2. Soziale Vorsorge als Modus der Gewährleistung sozialer Sicherheit

3. Soziale Sicherheit und Gleichheit: Vorsorge und Solidarität

III. Soziale Sicherheit durch Standardisierung sozialer Vorsorge

IV. Das „Vorsorgesystem“ des Grundgesetzes

B. Das „Vorsorgeverhältnis“ als rechtliches Gehäuse der Vorsorgebeziehungen

I. Verrechtlichung der Vorsorgebeziehungen

II. Soziale Vorsorge als Daseinsvorsorge

C. Vorsorge im Sozialstaat zwischen staatlicher und privater Verantwortung 72

I. Entwicklungspfad sozialer Vorsorge: Die „Sozialversicherung“

II. Vorsorgedefizite in der sozialen Wirklichkeit $\quad 74$

1. Prekäre Grundannahmen für „Sozialversicherung“

2. Soziale Sicherheit als erweitertes Vorsorgeziel 76

3. Das Gleichheits- und Gerechtigkeitsproblem egalitärer Vorsorge 77

D. Modernisierung der klassischen Vorsorgesysteme am Beispiel der gesetzlichen Kranken- und Rentenversicherung

I. Die Gesundheitsreform 2007 als „Systemwandel“

1. Systemwandel in der gesetzlichen Krankenversicherung

2. Merkmale des Systemwandels und „Wettbewerb“ als Steuerungskonzept

3. „Status-Passage“ für freie Berufe und gelenkter Vertragswettbewerb

4. Divergenzen zur herkömmlichen vertraglichen Konkretisierung von Rechtsnormen

5. Auf dem Weg zur Vereinheitlichung von öffentlicher und privater Krankenversicherung

6. Geltung des Wettbewerbs- und Kartellvergaberechts

II. Neue Architektur der gesetzlichen Rentenversicherung

1. Die gesetzliche Rentenversicherung als „Grundsystem“ der Altersvorsorge

2. Grundsicherung durch steuerfinanzierte Sockelrente?

3. Zukunftsdefizite der gesetzlichen Rentenversicherung 
4. Konzeptionelle Ansätze zu einer „,neuen“ Altersvorsorge:

Die Mehrsäulen-Strategie

5. Verfassungsrechtliche Grenzziehungen für die Modernisierung des Rentensystems

E. Art und Maß der zukünftigen Vorsorge als „Neue soziale Frage“

I. Vorsorge durch „Markt und Wettbewerb“ - oder:

Wer kann sich künftig welche Sicherung leisten? 91

II. Probleme der Verweisung auf private Vorsorgeverantwortung 92

III. Die Gegenposition: Keine „Verantwortungsteilung“ im Sozialstaat $\quad 94$

F. Struktur- und Funktionswandel des „Vorsorgeverhältnisses“

I. Wandel der funktionalen Perspektive 95

II. Strukturwandel des „Vorsorgeverhältnisses“ und staatliche Auffangverantwortung $\quad 97$

III. Staatliche Regulierung als Strukturkonsequenz 97

IV. Organisationsrechtliche Konsequenzen 99

G. Soziale Vorsorge im ausländischen und vergleichenden Kontext 99

I. Zwischenbilanz und Notwendigkeit der vergleichenden Perspektive 99

1. Unbehelflichkeit der nationalen Diskussion über die Entstaatlichung
sozialer Vorsorgeaufgaben

2. Entwicklung sozialer Vorsorge in vergleichender Perspektive 100

II. Ausländische und supranationale Leitbilder für Vorsorgemodernisierung 102

1. Die Niederlande als Leitbild deutscher Modernisierungsanstrengungen 102

2. Entwicklungslinien sozialer Sicherheit im Recht der Europäischen Union 103

3. Ein neuer Gesellschaftsvertrag für soziale Vorsorge und Sicherheit aus internationaler Perspektive?

4. Notwendigkeit eines dynamischen Rahmenkonzepts zur Förderung eigenverantwortlicher sozialer Vorsorge

\section{A. Sicherheit als Verfassungsprinzip und soziale Vorsorge als ihr Gewährleistungsmodus}

\section{Der Sozialstaat als Staatsziel}

„Zwei zentrale Aussagen“, so formuliert H.F. Zacher in seiner eindrucksvollen Abhandlung über „Das soziale Staatsziel“ im Handbuch des Staatsrechts der Bundesrepublik Deutschland (Bd. IV), würden den deutschen Sozialstaat in Europa und inmitten der Globalisierung prägen, nämlich die „Verantwortung des Sozialstaats für das Existenznotwendige“ einerseits, der „Primat der Selbstverantwortung“ andererseits; unter diesem habe sich das „Soziale ... wesentlich auch durch die Gesellschaft und in der Gesellschaft zu vollziehen“. ${ }^{1}$ Beide Verantwortungsstränge, die im Grundgesetz wurzelten, verhielten sich, so fährt er fort, zueinander in einer gewissen Dialektik, die in der Meta-

1 Zacher, Das soziale Staatsziel, in: Isensee/Kirchhof (Hrsg.), Handbuch des Staatsrechts, Bd. II, 3. Aufl. Heidelberg 2004, § 28 Rdnr. 31. 
pher vom „freiheitlichen Sozialstaat“" sinnfällig abgebildet werde. ${ }^{2}$ Zutreffend wertet der Jubilar diese Dialektik als „die entscheidenste Prämisse jeder interpretatorischen Entfaltung des Sozialstaatsprinzips".3

\section{Sicherheit als Verfassungsprinzip des Sozialstaats}

In diesem Verständnis leitet der Sozialstaat als Staatszielbestimmung des Grundgesetzes die um weitere verfassungsrechtliche Maßgaben ergänzte und gesetzlich detaillierte Ausgestaltung der Sozialordnung an. Rechtsstaatliche Freiheit sieht sich mit (sozialer) Sicherheit verschmolzen. ${ }^{4}$ Letztere umfasst die Gesamtheit der staatlichen Schutzversprechen, mit denen die Staatsfunktionen der Gesetzgebung, Verwaltung und Rechtsprechung das soziale Staatsziel unter Beachtung der grundgesetzlichen Freiheits- und Gleichheitsgarantien zu verwirklichen suchen. Beide, das Sicherheits- wie das Freiheitsprinzip bilden aus dieser Sicht ebenso Grund wie Grenze des Sozialstaats; darüber hinaus statuieren die Gleichheitssätze im „sozialen Rechtstaat“ (Art. 28 Abs. 1 GG) ein Grundmaß an sozialer Gerechtigkeit und sozialem Ausgleich. ${ }^{5}$

\section{Das Verfassungsprinzip ,,Sicherheit“}

„Sicherheit“" wird dadurch (nebst „Gleichheit“) für den Sozialsektor nicht nur als sozio-kulturelles Wertsymbol verankert. Sie gibt sich zugleich verfassungskonstitutionell unter Hinwendung zum sozialen Staatsziel als eigenständiges Verfassungsprinzip zu erkennen. Es macht die Unsicherheiten der gesellschaftlichen Lebensführung ebenso sehr wie die staatlichen Reaktionen hierauf und im Hinblick auf soziale Risiken von Verfassungs wegen zu einem eigenen Thema. ${ }^{6}$

Das Grundgesetz stabilisiert auf diese Weise das Vertrauen der Verfassungsbürger in die Schutzfähigkeit ihres Sozialstaates. Es vermittelt diese Erwartungssicherheit in der Deckung sozialer Bedarfe und es verbindet damit konkrete Rahmenmaßgaben für die staatliche Sicherheitsgewähr auch im Bereich der sozialen Sicherheit. Diese herbeizuführen, steht m. a. W. nicht im Belieben der Gesetzgebung. Sie wird statt dessen und jenseits der auf der Grundlage des Rechtsstaatsprinzips bereits verwirklichten rechtsförmlichen Ansprüche durch das Grundgesetz gefordert. Die Folge sind einerseits Min-

2 Zacher, a. a. O., Rdnr. 26; vgl. auch Pitschas, Die Zukunft der sozialen Sicherungssysteme, VVDStRL Bd. 64 (2005), S. 109 (112 f.).

3 Zacher, a. a. O., Rdnr. 26.

4 Pitschas (Fußn. 2), VVDStRL Bd. 64 (2005), S. 112 f., 113 ff.

5 Scholz/Pitschas, Sozialstaat und Gleichheit, in: Festschrift zum 25jährigen Bestehen des BSG, Bd. 2, 1979, S. 627 (642 ff., 666 ff.); Kirchhof, Der allgemeine Gleichheitssatz, in: Isensee/Kirchhof (Hrsg.), Handbuch des Staatsrechts, Bd. V, 2. Aufl. Heidelberg 2000, § 124 Rdnr. 19, 25, 29, 40.

6 Grdl. dazu Pitschas (Fußn. 2), VVDStRL Bd. 64 (2005), S. 113 ff., 136 f. 
destpflichten des sozialen Rechtsstaats, die sich im Mehr-Ebenen-System der Europäischen Gemeinschaft (EG) durch den Vertrag von Lissabon zu sozialen Verbürgungen verdichtet haben. ${ }^{7}$ Darüber hinaus geht es mitgliedstaatlich um anspruchsrechtlich geronnene Sozialstaatlichkeit; diese braucht Erfüllungsgewissheit. Die Antwort auf die Frage, wie sicher etwa Sozialversicherung ist, gibt das Grundgesetz daher auf einem doppelten Weg: Einerseits verweist es hierfür auf den rechtsstaatlichen und grundrechtlichen Vertrauensschutz. ${ }^{8}$ Auf der anderen Seite muss aber die Verfassung auch die Frage beantworten, wie soziale Sicherung (einschließlich der Sozialversicherung) unter den Herausforderungen aktueller Krisen künftig zu entwickeln ist. Dann steht nicht Bestandsschutz, sondern die Sicherung der Erwartungssicherheit im Vordergrund.

Diese schützt wiederum das Verfassungsprinzip Sicherheit des Sozialstaats, das Erwartungsenttäuschungen vermieden wissen will. Es fordert als Konsequenz aus dem Zusammenwirken mit dem Verfassungsgrundsatz der „sozialen Gleichheit“, dass im Verlauf der „sozialen Realisation“ (E. Forsthoff) die Gewissheit und das Vertrauen in staatliche Sicherheitsaktivitäten $\mathrm{zu}$ stabilisieren und damit delegitimierende Erwartungsenttäuschungen von Verfassungs wegen zu begrenzen sind.

\section{Soziale Vorsorge als Modus der Gewährleistung sozialer Sicherheit}

Das Verfassungsprinzip Sicherheit umgreift vor diesem Hintergrund auch Bestand und Reichweite der sozialen Vorsorge gegen die als „Wechselfälle des Lebens“ auftretenden sozialen Risiken der Krankheit, Invalidität, Alter, Arbeitslosigkeit u. a. m. Sie sind im Einzelfall mit dem Verlust des Einkommens oder des Unterhalts bzw. mit besonderen Aufwendungen wie etwa jenen für die Alterssicherung und oft auch für die medizinische Versorgung verbunden. Die vom Einzelfall abstrahierende, subjektiv berechtigende normative Zusage von Leistungen im Fall der Verwirklichung dieser Risiken bezeichnen wir als „soziale Vorsorge“, insofern diese zumindest dem Grunde nach - bei Renten aber auch wegen deren Lohnersatzfunktion der Höhe nach - unter rechtlicher und tatsächlicher Maßgabe des jeweiligen Vorsorgeversprechens steht. Dies schließt allerdings eine konkrete Bedarfsorientierung nicht immer aus. ${ }^{9}$

Allerdings lässt erst die staatliche Garantie, die mit dieser Vorsorge verbundenen Erwartungen auch tatsächlich zu erfüllen, individuelle Vorsorgeanstrengungen überhaupt als sinnvoll erscheinen. Erst sie ermöglicht auch dem Gesetzgeber der sozialen

7 Art. 6 Abs. 1 Vertrag über die Europäische Union (EU) nach der Änderung durch den Vertrag von Lissabon v. 13.12.2007, ABl. Nr. C 306/1 v. 17.12.2007; Art. 27 ff. Charta der Grundrechte der Europäischen Union v. 7.12.2000; ABl Nr. C 364/1.

8 Dazu näher mit Nachw. aus der Rspr. des BVerfG Papier, Der Einfluss des Verfassungsrechts auf das Sozialrecht, in: v. Maydell/Ruland/Becker (Hrsg.), Sozialrechtshandbuch (SRH), 4. Aufl. Baden-Baden 2008, § 3 Rdnr. 123 ff.

9 Zacher, in: Isensee/Kirchhof (Hrsg.), Handbuch des Staatsrechts (Fußn. 1), Rdnr. 45. 
Modernisierung, den Wandel sozialer Sicherung von der Versorgung zur Vorsorge mit deren privaten Anteilen strategisch zu vollziehen.

Umgekehrt fordert das Verfassungsprinzip Sicherheit dem Sozialbürger die Bereitschaft zur Vorsorge und deren Realisierung ab. Es setzt diese insoweit voraus. Andernfalls verlöre es sein Substrat. Darin liegt letztlich das Anerkenntnis des Primats der Eigenverantwortung für soziale Sicherung mit dem Ziel entsprechender Sicherheit. Zugleich bleibt für deren Gewährleistung der Zusammenhang mit der sozialen Gleichheit und der ihr ersprießenden Pflicht zur Wahrung von Solidarität zu beachten.

\section{Soziale Sicherheit und Gleichheit: Vorsorge und Solidarität}

Der prinzipienhaften Entfaltung von (sozialer) Sicherheit gesellt sich im Sozialstaat die soziale Gleichheit als komplementäres Verfassungsprinzip hinzu. Der Verfassungsstaat gewährleistet seinen Bürgern nicht nur, dass diese in körperlicher, technischer oder auch sozialer Sicherheit leben dürfen, sondern dass zudem Gleichheit in Sicherheit gewährleistet ist. Denn, wie H. F. Zacher formuliert hat, ist ,die Gleichheit aller, die Menschenantlitz tragen, ... der allgemeinste und tiefste Grund der sozialen Bewegung“. ${ }^{10}$ Die Vorstellung, dass soziale Gleichheit auch gesicherte Gleichheit erfordert, verknüpft deshalb Vorsorge mit Erwartungssicherheit und beruft Solidarität als Bürgen hierfür: Staat und Gesellschaft tragen dafür Verantwortung, dass ,generell Benachteiligungsgefährdete (nicht) - auch oder insbesondere - spezifisch sozial benachteiligt werden"“. ${ }^{11}$ Augenfällig ist das hinsichtlich des Verbots, Behinderte zurückzusetzen (Art. 3 Abs. 3 S. 2 GG). Das Spiel von Freiheit und Gleichheit wird durch diese verfassungsrechtliche Verpflichtung des Staates darauf eingerichtet, gleiche Sicherheit für alle zu bewirken.

Gleichwohl wissen wir, dass der sozialen Sicherung immanente Gleichheits- und Gerechtigkeitsprobleme bleiben. Diese sind zunächst und vor allem allgemeiner Art, insofern jede soziale Vorsorge in der Dialektik von sozialer Verantwortung des Staates auf der einen und der Versorgungsautonomie der Gesellschaft auf der anderen Seite steht. Mit der Freisetzung spezifischer sozialer Standards wie der Generationen- oder auch Beitragsgerechtigkeit versucht der Gesetzgeber, Gleichheit in sozialer Sicherheit zu realisieren.

\section{Soziale Sicherheit durch Standardisierung sozialer Vorsorge}

Es versteht sich von selbst, dass auf diesem Weg erreichte soziale Sicherheit keinen Endzustand aller Anstrengungen begründet. Ebenso wenig stellt sie sich gleichsam naturwüchsig ein. Sie bedarf staatlicher Anstrengungen. Inmitten des Prozesscharakters

10 Zacher, ebda. (Fußn. 1), Rdnr. 34.

11 Zacher, ebda. (Fußn. 1), Rdnr. 37. 
dieser ihrer Entfaltung unterliegt sie durch Dialog und Kommunikation im Zusammenhang gesetzgeberischer Veranlassung, sozialer Governance und institutioneller Brechung der Politikformulierung bzw. Rechtsdurchsetzung 12 einer schrittweisen Zielorientierung, Leitbildentwicklung, Konkretisierung und dabei Maßstabsetzung, kurz: der Ausformung sozialer Standards. Deren Verwirklichung kennzeichnet das angestrebte Sicherheitsniveau.

Die Erfüllung der in diesen Standards genormten Vorsorgeversprechen des Staates bzw. privater Vorsorgegeber trägt der Erwartungssicherheit auf Seiten der Sozialbürger Rechnung. Solche Standards weisen zugleich partizipatorischen Charakter durch ihre prozesshafte Entwicklung im Einklang mit Verbänden, Nichtregierungsorganisationen und anderen Institutionen der Zivilgesellschaft auf. In den europäischen Mitgliedstaaten gewinnen darüber hinaus soziale Vorsorgestandards, wie sich am Beispiel der Alterssicherung zeigt, sogar grenzüberschreitende Reichweite. ${ }^{13}$

Der aus alledem resultierende Prozesscharakter ihrer Entfaltung entspricht der Dynamik sozialer Sicherheit im Verfassungsstaat. Inhaltliche Festlegungen, wie die Maßgabe der Gleichbehandlung im Zugang zu Sozialleistungen oder auch der Existenz einer Grundsicherung als Konsequenz des Verfassungsprinzips Sicherheit nehmen sodann im einzelnen Rechtsverhältnis der sozialen Vorsorge rechtlich verbindliche Gestalt an. Dort konkretisieren sich auch allgemeine Verfahrensstandards wie etwa die Verpflichtung zur Kooperation von Sozialbehörden im Europäischen Verwaltungsverbund ${ }^{14}$ bzw. aus verwaltungswissenschaftlicher Perspektive das Benchmarking der von ihnen erbrachten Sozialleistungen. Standards der sozialen Vorsorge setzt nicht zuletzt die Auswahl der damit betrauten Institutionen einschließlich ihrer Differenzierung für die Gewährleistung von Vorsorgesicherheit, z.B. in Gestalt der Ausformung einer „Sozialversicherung".

\section{Das „,Vorsorgesystem“ des Grundgesetzes}

Dem Grundgesetz ist soziale Vorsorge in Gestalt der Sozialversicherung selbstverständlich (vgl. Art. 74 Abs. 1 Nr. 12, 87 Abs. 2, 120 Abs 1 S. 4 GG). Die Verfassung garantiert sie ferner im Wege der Beamtenversorgung (Art. 33 Abs. 4 und 5, Art. 74a GG). Darüber hinaus werden verfassungsrechtlich einzelne Tatbestände des sozialen

12 Pitschas, Nationale Gesundheitsreform und europäische „Governance“ der Gesundheitspolitik - Zur Verpflichtung der Gemeinschaftsstaaten auf solidarischen Wettbewerb durch „offene Koordination“, VSSR 2002, S. 75 ff.

13 Rische, Der Einfluss europäischer Sozialpolitik auf die gesetzliche Rentenversicherung, RVaktuell 2009, S. 2 ff.

14 Pitschas, Europäisches Verwaltungsverfahrensrecht und Handlungsformen der gemeinschaftsrechtlichen Verwaltungskooperation, in: Hill/Pitschas, Europäisches Verwaltungsverfahrensrecht, Berlin 2004, S. 301 ff., 319 f., 323 f., 329 ff. 
Entschädigungsrechts (vgl. Art. 74 Abs. 1 Nr. 6, 9, 10; Art. 119, 120, 120a GG) mit dem Vorsorgeauftrag verbunden.

Diese Wechselbezüglichkeit macht auf die heutigen Schwierigkeiten der systematischen Differenzierung sozialer Sicherung aufmerksam. Es scheint so, als ob ambitionierten Versuchen der „,systemischen“ Zuordnung von Risiken und Vorsorge zunehmend ein nur noch heuristischer Wert, aber keine entscheidende Funktion mehr für die marktorientierte Sozial- und Gesundheitspolitik zukommt. So lässt sich zwar in Abkehr von früheren Systematisierungen grosso modo die „Vorsorge“ von den sozialen Entschädigungssystemen und den Hilfs- und Förderungssystemen dogmatisch noch immer trennen. ${ }^{15}$ Im Wandel der Konzeption des öffentlichen Dienstes in Deutschland wird man allerdings nachfragen müssen, ob innerhalb des Vorsorgezusammenhangs nach dem Grundgesetz dem historisch gegenüber der „Sozialversicherung“ als Vorsorge(Teil-)system bestehenden älteren Grundtyp der Beamtenversorgung zukünftig noch ein Eigenleben als „Versorgungssystem“ zuzubilligen ist. Das dürfte eher und angesichts der Bezugnahmen des Gesetzgebers bei Reformschritten auf die gesetzliche Rentenversicherung (GRV), nämlich durch „wirkungsgleiche“ Abschmelzung von Renten- und dienstlichen Versorgungsansprüchen einen Gleichklang herzustellen, zweifelhaft sein. ${ }^{16}$ Aus alledem fällt schließlich der nicht systemfähige und nahezu ausschließlich politisch geleitete Strukturentwurf der Abgeordnetenversorgung gänzlich heraus.

\section{B. Das „, Vorsorgeverhältnis“ als rechtliches Gehäuse der Vorsorgebeziehungen}

In der Art und Weise, wie das Grundgesetz soziale Vorsorge als den zentralen Mechanismus der Gewährleistung sozialer Sicherheit für die Staatsbürger anlegt, spiegelt sich die durch den sozialen Rechtsstaat begründete Eigenart der genannten Vorsorgesysteme dienst- wie sozialversicherungsrechtlicher Art wider. Es setzt Vorsorgefähigkeit (Dienstleistungsfähigkeit, Beitragsfähigkeit) voraus und es stellt teilweise auf Vorsorgebedürftigkeit ab. Ziel der Vorsorge ist es, im Falle der Verwirklichung rechtlich beschriebener sozialer Risiken eine Vorsorgeleistung zu erbringen, die den Lebensstandard des Gesicherten aufrecht zu erhalten vermag.

In diesem Punkt unterscheidet sich soziale Vorsorge prinzipiell und einerseits von der Sicherung eines menschenwürdigen Existenzminimums für jedermann durch Sozialhilfe, andererseits aber auch von einer immer stärker diskutierten Grundsicherung im

15 Vgl. den „Systemüberblick“ bei Igl/Welti, Sozialrecht, 8. Aufl. Düsseldorf 2007, § 2 Rdnr. 3; ferner Eichenhofer, Zum System des Sozialrechts, SGb 1998, S. 289 ff.

16 S. auch Hase, Rentenversicherung und Beamtenversorgung, in: Becker/Kaufmann/v. Maydell/Schmähl/Zacher (Hrsg.), Alterssicherung in Deutschland, Festschrift für Franz Ruland zum 65. Geburtstag, Baden-Baden 2007, S. 495 ff. in Auseinandersetzung mit BVerfGE 114, 258. 
Alter. ${ }^{17}$ Vorsorge erfüllt zwar auch einen elementaren sozialstaatlichen Zweck der Abwehr von Not, geht aber darüber hinaus; sie verspricht sichere Leistungen für diejenigen, die durch Beiträge oder auf andere Art für ihre Lebensstandardsicherung in Risikolagen haben „vorleisten“ können bzw. für die von dritter Seite vorgeleistet wurde.

\section{Verrechtlichung der Vorsorgebeziehungen}

Dieses „Vorsorgeverhältnis“ im Sozialrecht 18 bildet das rechtliche Gehäuse für die mit den voraufgehend skizzierten Zielen ausgestatteten und in rechtlichen Anspruchsnormen institutionell geronnenen sowie sozial- wie rechtsstaatlich gewährleisteten Vorsorgeversprechen staatlicher Sozial- und Gesundheitspolitik, die soziale Sicherheit ermöglichen will und von Verfassungs wegen dies auch muss. Seine Einkleidung als „Rechtsverhältnis“" gestattet es, soziale Vorsorge als abstrahierende, im Einzelfall dann aber subjektiv berechtigende normative Zusage von Leistungen im Falle einer typischen Risikosituation auf der Grundlage durchgestalteter und nicht abhandelbarer Gegenseitigkeitsbeziehungen einzurichten. Zutreffend ist deshalb darauf hingewiesen worden, dass soziale Vorsorge als Teil der ,sozialen Sicherheit" auf diese Weise auch Rechtssicherheit verkörpert, somit der verfassungsrechtlichen Verbindung von Rechts- und Sozialstaat Ausdruck gibt. 19

Zugleich aber und darüber hinausgehend verleiht das Vorsorgeverhältnis dem ihm zugrundeliegenden Sicherheitsgedanken eine überschießende und eigenlegitimierte differenzierte Bedeutung: Indem soziale Vorsorge für den typischen Bedarfsfall das - nach Maßgabe ihrer Ausgestaltung - typisierte ,richtige“ verspricht, erscheint sie als gesicherte Erwartung; sie verkörpert „Sicherheit“ gleichsam i. S. eines unbedingten Versprechens. Daraus erhellt, dass neben dem Rechtsstaat und seinen grundrechtlichen Garantien für soziale Freiheit auch noch andere Wurzeln die soziale Vorsorge verfassungsrechtlich nähren. $\mathrm{Zu}$ ihnen gehört das bereits erörterte Verfassungsprinzip „Sicherheit".20

17 Zur Diskussion s. zul. etwa Ruland, Die Zukunft der Alterssicherung aus heutiger Perspektive, SGb 2008, S. 570 (574 f.); aus verfassungsrechtlicher Sicht Jährling-Rahnefeld, Verfassungsmäßigkeit der Grundrente, Frankfurt a.M. 2002, bes. S. 82 ff., 94 ff., 213 ff.

$18 \mathrm{Zu}$ dessen Begriff, dogmatischen Grundlagen und Reichweite s. auch Eichenhofer, Sozialrecht, 6. Aufl. Tübingen 2007, § 12 Rdnr. 268 ff., § 13 Rdnr. 289 f.; Fuchs/Preis, Sozialversicherungsrecht, Köln 2005, § 41 I und II (S. 641 ff.).

19 Zacher, in: Isensee/Kirchhof (Hrsg.), Handbuch des Staatsrechts (Fußn. 1), Rdnr. 45 m. Anm. 252.

20 In diese Richtung auch Rüfner, Daseinsvorsorge und soziale Sicherheit, in: Isensee/Kirchhof (Hrsg.), Handbuch des Staatsrechts, Bd. IV, 3. Aufl. Heidelberg 2006, § 96 Rdnr. 83, vgl. ferner Rdnr. 22: „Staatsaufgabe ist die Sicherung der Versorgung“. 


\section{Soziale Vorsorge als Daseinsvorsorge}

Das Ziel der sozialen Vorsorge, bei Eintritt ihrer Notwendigkeit die zuvor zugesicherte und vertypte Leistung zu erhalten, sich dieser also „,sicher“ sein zu dürfen, begründet einen inneren Zusammenhang mit dem übergreifenden sozialstaatlichen Konzept der Daseinsvorsorge. ${ }^{21}$ Jedenfalls wirft der gleichheitsdirigierte Auftrag des Sozialstaats, auch im Rahmen der Vorsorge für sozialen Ausgleich zu sorgen und dabei insbesondere auf die Vorsorgebedürftigkeit zu achten, die Frage auf, wie sich der Sozialstaat und die Daseinsvorsorge zu einander verhalten. Sich dessen zu vergewissern, ist wegen der aller Daseinsvorsorge in der Europäischen Gemeinschaft anhaftenden Verbindungslinien zum entstehenden Europäischen Sozialstaat als eigenständigem Verfassungsgrund neben dem Binnenmarkt (etwa: Art. 86 II EGV) um so mehr unverzichtbar, als die Gemeinschaft nunmehr und auf der Grundlage des Vertrags von Lissabon einen neuen Anlauf für ein eigenes Konzept der „Daseinsvorsorge“ unternommen hat. Darüber hinaus unterliegen Versorgungsaufgaben im Rahmen der Daseinsvorsorge besonderen Modalitäten im Rahmen der allgemeinen Privatisierung. ${ }^{22}$

Die übliche Antwort auf das Verhältnis von sozialer Sicherung zur Daseinsvorsorge will zwischen Leistungen dieser und Sozialleistungen einschließlich sozialer Vorsorge deutlich unterscheiden. Während nämlich Daseinsvorsorge die Sicherung der allen Bürgern gleichmäßig anzubietenden Versorgung mit öffentlichen Gütern anstrebe, so heißt es, richteten sich soziale Vorsorge bzw. der soziale Auftrag darauf, dass nicht sozial Schwächere benachteiligt oder ausgeschlossen würden. Hierin realisiere sich die sozialstaatliche Gleichheitsverantwortung. Dementsprechend sei Daseinsvorsorge dann „,sozial“, so wird argumentiert, ,wenn und soweit sie durch die allgemeine Vorhaltung und Darreichung gemeinwohlorientierter Dienste und Güter die Bedeutung des sozialen Gefälles in der Gesellschaft für den Zugang zu diesen und öffentlichen Diensten“ entschärfe. 23

Freilich hebt die allgemeine Bereitstellung von gemeinwohlbezogenen Gütern und Diensten wie z.B. von Sach- und Dienstleistungen der öffentlichen Hand für die Allgemeinheit die Ungleichheit der individuellen Vorsorgefähigkeit noch nicht auf. Zutreffend sehen daher Einrichtungen des Staates bzw. der Kommunen Leistungen in Krankenhäusern, Heimen, von Sozialstationen oder Rettungsdiensten als solche der Daseinsvorsorge vor. Diese „sozialen Dienste“ weisen eine soziale Komponente im Sinne des sozialen Ausgleichs auf, weil die Versorgung zu tragbaren Bedingungen für alle bereitgehalten wird.

21 Rüfner, in: Isensee/Kirchhof (Hrsg.), Handbuch des Staatsrechts (Fußn. 20), Rdnr. 13.

22 Pitschas, Kommunale Daseinsvorsorge im europäischen Binnenmarkt - Grenzen der Liberalisierung kommunaler Dienstleistungen in der europäischen Wettbewerbsgesellschaft, in: ders./Ziekow (Hrsg.), Kommunalwirtschaft im Europa der Regionen, Berlin 2004, S. 33 (44 ff.).

23 Zacher, in: Isensee/Kirchhof (Hrsg.), Handbuch des Staatsrechts (Fußn. 1), Rdnr. 66. 
Beispiele wie diese zeigen, dass zwischen Versorgungsaufgaben der Daseinsvorsorge und Leistungen der sozialen Sicherung i. S. der sozialrechtlichen Vorsorge (im formellen Sinne) nicht strikt zu trennen ist. Nicht von ungefähr hat daher schon E. Forsthoff die Leistungen der Rentenversicherung in eine Reihe mit anderen Leistungen der Daseinsvorsorge wie etwa der Verfügbarkeit von Verkehrsmitteln und der Wasserversorgung gestellt. Heute finden wir aus der skizzierten inneren Gemeinsamkeit von Daseinsvorsorge und sozialer Vorsorge resultierende Probleme vor allem in der Krankenhausversorgung und -finanzierung, aber auch in der Prävention einerseits, in der Rehabilitation andererseits. Nicht zuletzt an ihnen wird deutlich, wie bereits $H$. F. Zacher festgestellt hat, dass sich Sozialpolitik auf der einen Seite und Daseinsvorsorge auf der anderen Seite zumindest i. S. zwei sich überschneidender Kreise begegnen. ${ }^{24}$

Sie gehen jedoch wegen der beide überwölbenden Aufgabe der Gemeinwohlsicherung nicht einmal wirklich dort auseinander, wo die Priorität der Daseinsvorsorge in der Allgemeinheit der Versorgung liegt, die Sozialpolitik aber bedacht darauf nehmen muss, allfällige Unterschiede im Zugang zu Dienstleistungen und Gütern auszugleichen, die auf den sozialen Verhältnissen der Nachfrager beruhen. Vielmehr verschmelzen beide miteinander im Auftrag der Sicherstellung optionaler Güter bei der Vorsorge gegen soziale Risiken, mag es um die Armutsfestigkeit der Alterssicherung oder um die Erschwinglichkeit der Wasserversorgung für gering bemittelte Bürger zu sozialen Tarifen in Kommunen gehen.

\section{Vorsorge im Sozialstaat zwischen staatlicher und privater Verantwortung}

Bei alledem unterliegen auch die Vorsorgeleistungen der sozialen Sicherung inmitten der Versorgungsaufgaben aller Daseinsvorsorge dem Wandel zum sog. Gewährleistungsstaat. Zwar ist die Daseinsvorsorge eine diskretionäre Aufgabe. Ob allerdings damit auch die „Privatisierung“ der sozialen Sicherung bzw. Vorsorge dem Ermessen des Gesetzgebers und der Verwaltung - wie z.B. in der gesetzlichen Krankenversicherung (GKV) dem Gemeinsamen Bundesausschuss (G-BA) - überlassen werden darf, ist eine andere Frage. Ich zweifle daran: Die Modernisierung der sozialen Vorsorge darf in Deutschland und europaweit nicht nach Maßgabe einer sog. Verantwortungsteilung die in anderen Sachbereichen gewählten Modalitäten entstaatlichter Sicherstellung auf den Bereich der sozialen Vorsorge übertragen. Das Konzept des sog. Gewährleistungsstaates, also eines Staates, der soziale Vorsorge nicht mehr selbst organisiert, sondern sich wie partiell bei der privaten Altersvorsorge - auf die Gewährleistung ihrer gesellschaft-

24 Zacher, in: Isensee/Kirchhof (Hrsg.), Handbuch des Staatsrechts (Fußn. 1), Rdnr. 67. 
lichen Angebote zurückzieht, ${ }^{25}$ wird jedenfalls durch das Verfassungsprinzip Sicherheit modifiziert. Der Staat darf die staatliche Verantwortung für die Sicherung der Vorsorge nicht aufgeben, indem er sie ,aufteilt“, d. h. Anteile daran dem freien Spiel der Marktkräfte, etwa in der gesetzlichen Krankenversicherung, in Kernbereichen überlässt. Der Verweis auf eine insofern fortbestehende „Auffangverantwortung“ geriete dann lediglich zu einem semantischen Wortspiel. Die Sicherung der Versorgung ist vielmehr eine staatliche Kernaufgabe. ${ }^{26}$ Infolgedessen gilt es, auf dem Referenzgebiet der Vorsorge und ergänzend zu der Subsidiarität des Sozialstaats stärker nach den Folgewirkungen der sog. Verantwortungsteilung zu fragen, also die Notwendigkeit und Eignung des Gewährleistungskonzepts bereichsweise zu überprüfen.

Die Bedeutung des Themas spiegelt sich - wie bereits angemerkt - in der ausgreifenden Einwirkung der Europäischen Union (EU) auf die soziale Vorsorge in den Mitgliedstaaten wider: Weder ist der „Sozialstaat“ zukünftig allein ein „,nationalstaatliches Projekt", noch hat die gemeinschaftsrechtliche Modernisierung des Sozialschutzes in der EU ihr Ende erreicht. Ganz im Gegenteil belegt der Blick auf den Krankenversicherungssektor, dass auf einem entstehenden Gemeinsamen Markt für Sozial- und Gesundheitsleistungen die inzwischen revidierte „Unternehmensrechtsprechung“ des EuGH zum Status von gesetzlichen Krankenkassen unter ordnungspolitischen und wettbewerbsrechtlichen Aspekten von neuem aufbrechen dürfte und dann die Fragen nach der Reichweite der Art. 16, 86 II EGV entscheidende Bedeutung erlangen werden. ${ }^{27}$

\section{Entwicklungspfad sozialer Vorsorge: Die „,Sozialversicherung“}

$\mathrm{Ob}$ und wie sich auch soziale Vorsorge im Gewährleistungsstaat verändert, wird nicht nur durch ihre Einbettung in die staatliche Modernisierungspolitik beeinflusst. Erkenntnisse über den Wandlungsbedarf setzen ebenso und zunächst den (kursorischen) Blick auf ihren Entwicklungspfad voraus.

Die Entwicklung der sozialen Vorsorge in Deutschland mit der ihr innewohnenden rechtlichen Verfestigung eines spezifischen Vorsorgeverhältnisses lenkt zu allererst den Blick auf den Sozialstaat als einen (niemals abgeschlossenen) Prozess: Der Aufbau der sozialen Vorsorge bedeutet einen wesentlichen Schritt in dessen phasenhaften Verlauf. Denn auf der Grundlage einer langen Entwicklungsgeschichte im 19. Jahrhundert hat sich als Grundtyp sozialer Vorsorge die Sozialversicherung ausgeprägt, wie sie im Gefolge der Kaiserlichen Botschaft von 1881 entstanden ist. ${ }^{28}$ Die dieser inhärente politi-

25 Dazu m. w. Nachw. Voßkuhle, Neue Verwaltungsrechtswissenschaft, in: Hoffmann-Riem/SchmidtAßmann/Voßkuhle (Hrsg.), Grundlagen des Verwaltungsrechts, Bd. I, München 2006, § 1 Rdnr. 41.

26 Vgl. BVerfGE 115, 25 (43).

27 Ein Ansatz hierzu liegt in der geplanten „Patienten-Richtlinie“ der EU, vgl. Schulte Westenberg, Gesundheitsdienstleistungen in der EU, NZS 2009, S. $135 \mathrm{ff}$.

28 Näher noch Hase, Versicherungsprinzip und sozialer Ausgleich, Tübingen 2000, bes. S. $10 \mathrm{ff}$. 
sche ratio, nämlich für die Einrichtung des darin angekündigten Vorsorgesystems eine „Ansparphase“ hinsichtlich der Vorsorgeleistungen vorzusehen, die auf regelmäßiger Beitragszahlung im Rahmen eines Erwerbs- bzw. Beschäftigungsverhältnisses beruhen, um dann im Anschluss hieran den Leistungseintritt im Versorgungsfall zu finanzieren, entspricht der Grundannahme des Sozialrechts, wonach jeder Erwachsene die Möglichkeit hat und darauf angewiesen ist, den Lebensunterhalt für sich und seine Familie durch abhängige oder selbständige Arbeit zu verdienen.

Erforderlich ist dazu allerdings, dass aus dem Einsatz der Arbeitskraft ein hinreichendes Einkommen erzielt wird und darüber hinaus der Versicherte in der Lage ist, aus diesem Einkommen seinen Bedarf und den des jeweiligen Unterhaltsverbands zu decken. Einkommen kann dabei durch Vermögen ergänzt bzw. ersetzt werden. In der Regel aber müssen Arbeit und Einkommen gesichert sein. Schon dieser Hinweis reicht aus zu verdeutlichen, dass - wie H. F. Zacher zu Recht hervorgehoben hat - die „Grundregel“, wonach „Arbeit Einkommen erbringt und dieses der Befriedigung der Bedarfe auch im Unterhaltsverband dient", in ihrer Verwirklichung auf Grenzen stößt. Daraus entwickeln sich soziale Defizite, die sozialrechtliche Interventionen erforderlich machen. ${ }^{29}$

Dem Grunde nach kennzeichnet dieses aus der vorkonstitutionellen Entwicklung überkommene Trennungsmodell von Beschäftigungsverhältnis und Beitragszahlung einerseits, sozialem Vorsorge- und Leistungsverhältnis (Sozialversicherungsverhältnis) andererseits auch heute noch das Fundament der sozialen Vorsorge. Denn unter dem Sicherheits- und Schutzdispositiv des modernen Sozialstaats, der konstitutionell die soziale Vorsorge als „Sozialversicherung“ versteht (Art. 74 Abs. 1 Nr. 12 GG) und davon die Versorgung, Entschädigung, Hilfe und Förderung abtrennt, sieht sich das ,alte“ Vorsorgeverhältnis bis hin zur Einordnung sozialrechtlicher Anwartschaften in den Schutz der Kompetenzvorschriften und Grundrechte gestellt sowie von der Sicherheit als Verfassungsprinzip des Sozialstaats überwölbt.

\section{Vorsorgedefizite in der sozialen Wirklichkeit}

\section{Prekäre Grundannahmen für ,,Sozialversicherung“}

Die realempirische Entwicklung der sozialen Vorsorge offenbart allerdings seit mehr als einem Jahrzehnt den Übergang in eine ,neue“ Phase der Sozialstaatlichkeit. Mit ihrem Anbruch werden nicht nur die „Modalitäten der Aufgabenerfüllung“ (Rüfner) verändert. Vor dem Hintergrund der Globalisierung, weltweit demografischen Herausforderungen und daraus resultierender Ergänzungsbedarfe der sozialen Vorsorge einerseits, in Anbetracht wachsender Ungleichheit in der individuellen Fähigkeit, gegenüber sozia-

29 Zacher, in: Isensee/Kirchhof (Hrsg.), Handbuch des Staatsrechts (Fußn. 1), Rdnr. 28, 53 ff. 
len Risiken hinreichend vorzusorgen und entsprechend zugrundeliegender Armutsentwicklung sowie sozialer Gerechtigkeitsdefizite andererseits kommt es vielmehr zu einem prinzipiellen Widerstreit zwischen den Grundannahmen des Sozial(versicherungs) rechts und ihrer Verwirklichung. Die Folge ist - wie namentlich in der Krankenversicherung und Rentenversicherung erkennbar - der Rückschnitt der staatlichen Vorsorgeleistungen, der (ergänzende) private Vorsorge erforderlich macht. In der Tendenz sieht sich dadurch der Vorsorgecharakter der Sozialversicherung in Frage gestellt; sie wird staatlicherseits letztlich auf eine (beitrags- bzw. steuerungsfinanzierte) verpflichtende Grundsicherung zurückgenommen. ${ }^{30}$ Die jüngsten Vorschläge auf dem 67. Deutschen Juristentag für ein modernisiertes Arbeits- und Sozialrecht in der alternden Gesellschaft unterstützen sogar diese zweischneidige Entwicklung - ohne sich mit ihren „Schattenseiten" auseinanderzusetzen. ${ }^{31}$

Dazu sei an dieser Stelle nur soviel angemerkt: Dem grundgesetzlich konstitutionalisierten Vorsorgeverhältnis ist die Verpflichtung auf „soziale Sicherheit“" unverbrüchlich eingeschrieben. Denn es übersetzt die dem Sozialstaat material, kompetenziell und institutionell sowie als Staatsziel aufgegebene Sorge für sichere Versorgung und soziale Gerechtigkeit auf die individuelle Vollzugsebene dieser Versprechen. Dabei „Sicherheit“ in der Sache zu gewährleisten, also über die bloße „Rechtssicherheit“ hinausgehend auskömmliche Versorgung zu garantieren, ist der Gehalt des sozialstaatlichen Sicherheitsprinzips. Darin vollendet sich die grundgesetzliche Verbindung des sozialen Staatsziels mit dem Rechtsstaat. Die ehedem institutionalisierte „Sozialversicherung“ hat diese Aufgabe zwar lange Zeit erfolgreich wahrnehmen können. Sie versteht sich noch heute zu recht als Sicherung des erreichten Lebensstandards durch Bezugnahme der in sie zu entrichtenden Beiträge auf das jeweilige individuelle Einkommen. Die Beitragsbezogenheit der Leistungen überträgt dabei das Einkommensniveau auf das Leistungsniveau. Inzwischen unterliegt sie allerdings mit ihren festgefügten Strukturvorgaben und als mehr oder weniger tradierte Organisation sozialer Vorsorge weitreichenden Modernisierungsdefiziten. ${ }^{32}$

Ihre Grundannahmen sind zudem brüchig geworden. Zu Recht hat deshalb das BVerfG den entsprechenden Kompetenztitel in Art. 74 Abs. 1 Nr. 12 GG sukzessive geöffnet. System, Organisation und die sog. Selbstverwaltung der Sozialversicherung sind folgerichtig nicht verfassungsrechtlich ,geschlossen“. Der Umstand, dass nur eine dem Gattungsbegriff der „Sozialversicherung“ entsprechende Vorsorgeform auf die

30 Schmähl, Alterssicherungspolitik im Wandel, in: Becker/Kaufmann/v. Maydell/Schmähl/Zacher (Hrsg.), FS für F. Ruland (Fußn. 16), S. 291 (307 ff.).

31 Preis, Alternde Arbeitswelt - Welche arbeits- und sozialrechtlichen Regelungen empfehlen sich zur Anpassung der Rechtsstellung und zur Verbesserung der Beschäftigungschancen älterer Arbeitnehmer? Gutachten B für den 67. DJT, 2008, S. B 68 f., 109.

32 Vgl. Pitschas (Fußn. 2), VVDStRL Bd. 64 (2005), S. 125 ff.; Preis, ebda. (Fußn. 31), fügt dieser Einschätzung keine neueren empirischen Erkenntnisse hinzu; die von ihm erörterten Vorschläge zur Krisenbewältigung sind bekannt. 
Bundeskompetenz aus Art. 74 Abs. 1 Nr. 12 GG gestützt werden darf, 33 untersagt zugleich keineswegs - wie sich an der sozialrechtlichen Regulierung des privatrechtlichen Versicherungswesens zeigt ${ }^{34}$ - ihre Reichweite auszudehnen oder auch andere Formen der sozialen Vorsorge einzuführen. Mag auch die unlängst im Vorfeld des schon erwähnten Juristentages gebrauchte Formulierung, „das ... Sozialversicherungssystem geht von Axiomen aus, die (schon lange) nicht mehr stimmen", 35 ihrerseits nur partiell zutreffen, so erscheint daran jedenfalls richtig, dass mit der impliziten Bindung des „Vorsorgeverhältnisses“ an Beiträge auf der Grundlage möglichst lebenslang unbefristeter Vollzeitbeschäftigung und der Zielsetzung eines Ertrags umfassender Gesundheitsversorgung bzw. lebensstandardsichernder gesetzlicher Alterssicherung bei Erreichen der Regelaltersgrenze das Sozialversicherungssystem auf Konstruktionspfeilern errichtet worden ist, die vor dem Einsturz stehen.

\section{Soziale Sicherheit als erweitertes Vorsorgeziel}

Das internationale Recht hat deshalb zutreffend und in Abkehr von einer der „Versteinerung" ausgesetzten Systemsicherung sowie i. S. eines dynamischen Schutz- und Sicherungskonzepts den Begriff der Sozialversicherung weitgehend hinter sich gelassen. Es nutzt den weiteren Begriffsrahmen der „Sozialen Sicherheit“, wodurch in den Mittelpunkt auch der Vorsorgebestrebungen der abstrakt zugesagte soziale Schutz gegenüber typischen sozialen Risiken bei gleichzeitigem Versprechen der Absicherung ihnen gegenüber rückt. Zutreffend merkt $H$. F. Zacher hierzu an, dass dem vorzugwürdigen Verständnis von „Sozialer Sicherheit“ die „mit dem Begriff Sozialversicherung einhergehenden versicherungstechnischen Implikationen (Aufbau einer Anwartschaft in einem Versicherungsverhältnis; Finanzierung durch Beiträge; Selbstverwaltung) unwichtig" seien. 36

Diese Sichtweise ermöglicht in der Tat eher die Modernisierung sozialer Sicherheit vor dem Hintergrund bestehender oder eingetretener Sicherungsdefizite als das engere institutionelle Denken zur überkommenen „Sozialversicherung“. Denn dadurch darf der Gedanke der „Regulierung durch Selbstregulierung“, d. h. der Betätigung der Eigenverantwortung zur Vorsorge zukünftig signifikant hervortreten. Dem entspricht zugleich die Betonung steuerfinanzierter Hilfs- und Fördersysteme in den letzten Jahrzehnten. Zugleich wird das grundgesetzliche Postulat eines freiheitssichernden Sozialstaates gestärkt. Er ermöglicht Vorsorge, wie schon betont, durch eigene Anstrengungen im Wege

33 Vgl. etwa BVerfGE 75, 108 (146); 87, 1 (34); 88, 203 (313); 103, 97 (215); 114, 196 (221).

34 BVerfGE 103, 197 (215 ff.); Pitschas, Sozialrechtliche Regulierung des privatrechtlichen Versicherungswesens, in: Kluth/Müller/Peilert (Hrsg.), Wirtschaft - Verwaltung - Recht, Festschrift für Rolf Stober, Köln 2008, S. 295 (296 ff.).

35 Preis, Ein modernisiertes Arbeits- und Sozialrecht für eine alternde Gesellschaft, Beilage zu NJW Heft 21/2008, S. 9 r. Sp.; ähnlich bereits Pitschas (Fußn. 2), VVDStRL Bd. 64 (2005), S. 125 ff.

36 Zacher, in: Isensee/Kirchhof (Hrsg.), Handbuch des Staatsrechts (Fußn. 1), Rdnr. 46. 
der Vermögensbildung oder privaten Altersvorsorge bzw. Krankenversicherung. Hierbei steht vor allem der Rückbezug auf die Privatversicherung und damit auf die dem Grundgesetz vertraute duale Versicherungsverfassung im Vordergrund, die selbstverständlich dem Gesetzgeber einen weiten Spielraum zu legislativen Ausgestaltung belässt. ${ }^{37}$ Doch wäre auch eine pflichtige „Bürgerversicherung“ zur Förderung allgemeiner Vorsorge zulässig. ${ }^{38}$

Das künftige Recht der sozialen Sicherheit einschließlich der ihm erfließenden Vorsorgeanstrengungen sollte indes nicht mehr allein an den Beschäftigungsstatus anknüpfen. Darüber hinaus ist in der sozialen Wirklichkeit die gesetzliche Rentenversicherung längst keine Lebensstandardsicherung mehr und auch die Krankenversicherung nimmt Schritt für Schritt von einer vollumfänglichen Gesundheitsversorgung immer stärker Abstand. Hinzu kommt, dass die Sozialversicherung in ihrer herkömmlichen Konstruktion die Ungleichheit der Einkommen fortschreibt. Nimmt aber die strukturelle Arbeitslosigkeit zu und verlieren Einkommen als Ausdruck der Arbeitsleistung und Beiträge als Vorleistung sowie als Anteil an den Leistungen gegenüber anderen Versicherungsteilnehmern zugunsten steuerlicher Finanzierung immer mehr ihre Bedeutung, so bedarf soziale Vorsorge einer anderen Konstruktion, die geeigneter ist, sowohl den Problemen mangelnder Vorsorgefähigkeit als auch mangelnder Vorsorgebedürftigkeit entgegenzutreten. Freilich sollte auf die Beitragsbezogenheit der Vorsorge aus verfassungsrechtlichen Gründen auch in Zukunft nicht völlig verzichtet werden. 39

\section{Das Gleichheits- und Gerechtigkeitsproblem egalitärer Vorsorge}

Soziale Vorsorge steht inmitten der Dialektik sozialer Verantwortung des Staates auf der einen und der Versorgungsautonomie der Gesellschaft auf der anderen Seite von Anfang an sowie ungeachtet des Einbezugs immer breiterer Bevölkerungskreise und wachsender Steuerfinanzierung vor einem Gleichheits- und damit Gerechtigkeitsproblem. Obwohl die Grenze zwischen Vorsorgefähigkeit und Nicht-Vorsorgefähigkeit zumal in der Alterssicherung wesentlich ,nach unten“ verlagert worden ist, wird die verfassungsrechtlich aufgegebene soziale Gleichheit der sozialen Sicherung dort verfehlt, wo beispielsweise der Zugang zur Erwerbsarbeit nicht gewährleistet ist. Denn vorsorgefähig ist regelmäßig und in vollem Umfang nur der, der in einem Beschäftigungsverhältnis steht.

Die Krise der Arbeitsgesellschaft zeitigt daher tiefgreifende Konsequenzen für die soziale Sicherung und insbesondere die Arbeitsförderung einerseits wie die Altersvor-

37 Vgl. Pitschas, in: Kluth/Müller/Peilert (Hrsg.), FS für R. Stober (Fußn. 34), S. 298 ff.

38 Nachw. zur Diskussion um die „Bürgerversicherung“ im Einzelnen bei Rüfner, in: Isensee/Kirchhof (Hrsg.), Handbuch des Staatsrechts (Fußn. 20), Rdnr. 102 m. Anm. 287, 279.

39 Ebenso Krupp, Tendenzen der wirtschaftlichen Entwicklung und ihre Konsequenzen für die Alterssicherung, in: Becker/Kaufmann/v. Maydell/Schmähl/Zacher (Hrsg.), FS für F. Ruland (Fußn. 16), S. 171 (180 f., 183 ff.). 
sorge andererseits. Das Risiko, sich nicht durch Vorsorge gegen die primären sozialen Risiken (Krankheit u. a.) sichern zu können, stellt sich deshalb als ein weiteres, „sekundäres" soziales Risiko dar, demgegenüber die Sozialpolitik zwar spezifische Bewältigungsformen wie etwa die Anrechnung von Ersatz- und Ausfallzeiten in der Rentenversicherung oder die Zahlung von Rentenversicherungsbeiträgen im Rahmen von Einkommensersatzleistungen der GKV entwickelt hat. Allerdings werden dadurch die Probleme mangelnder Vorsorgefähigkeit in der Langzeitperspektive erfahrungsgemäß nicht entschärft.

Im Einzelnen sind es vier prinzipielle Gleichheitsdefizite, die näher zu entfalten wären. ${ }^{40}$ Neben der Ungleichheit im Zugang zur Erwerbstätigkeit (und dann eben auch bei der Beitragszahlung zur Sozialversicherung) bleibt zweitens die Leistungsdifferenzierung im Fall der Risikoverwirklichung durch die Vorsorge, weil sich das Einkommensniveau auf das spätere Leistungsniveau spiegelbildlich überträgt und dadurch die Ungleichheit der Einkommen fortgeschrieben wird. Hier werden Grenzen einer sozialstaatlichen Umverteilungspolitik mit der Folge eines nicht auflösbaren Spannungsverhältnisses zum sozialen Ziel höherer Gleichheit in der Gesellschaft erkennbar.

$\mathrm{Zu}$ den beiden weiteren Gleichheitsproblemen überkommener sozialer Vorsorge rechnet einerseits die mangelnde individuelle Bedarfsgerechtigkeit der Vorsorgeleistungen. Diese müssen kraft ihrer generalisierenden Auslegung den individuellen Bedarfsfall verfehlen; sie schaffen Ungleichheit durch Typisierung der sozialen Vorsorge. Daran wird allerdings auch zukünftig und zumal in der Krise der Beschäftigungsverhältnisse nur schwer etwas zu ändern sein. Schließlich und andererseits geht es in der sozialen Vorsorge darum, Vorsorgebedürftigkeit und Vorsorgefähigkeit wie z.B. in der Alterssicherung gruppentypisch zu bestimmen, also für Beschäftigte einerseits und freie Berufe oder auch Rentner („,Rehabilitation“) andererseits. Dadurch werden die einem persönlichkeitsrechtlichen Strukturkonzept des Grundgesetzes für die Lebenslage „Alter“41 erfließenden Direktiven einer verfassungsbestimmten Differenzierung in dem Verhältnis von sozialstaatlicher Vorsorgezuständigkeit, individueller Eigenverantwortung, Solidarität und Subsidiarität untereinander verfehlt - ganz abgesehen von weiteren Gleichheitsproblemen der Vorsorge und davon, dass die soziale Rentenversicherung schon heute für Höherverdienende lediglich eine Art „Grundsicherung“ darstellt und schon von daher Gleichheitsdefizite strukturbedingt existieren. ${ }^{42}$

40 Hierzu und im Folgenden auch Zacher, in: Isensee/Kirchhof (Hrsg.), Handbuch des Staatsrechts (Fußn. 1), Rdnr. 47 f.

41 Entfaltet bei Pitschas, Zur rechtlichen Verfassung der Lebenslage „Alter“, in: Gitter (Hrsg.), Festschrift für Otto Ernst Krasney zum 65. Geburtstag, München 1997, S. 355 (365 ff.).

42 Dazu und zu den daraus freigesetzten Legitimationsproblemen der staatlichen Alterssicherung Schmähl, in: Becker/Kaufmann/v. Maydell/Schmähl/Zacher (Hrsg.), FS für F. Ruland (Fußn. 30), S. 303, 309. 


\section{Modernisierung der klassischen Vorsorgesysteme am Beispiel der gesetzlichen Kranken- und Rentenversicherung}

Abhilfe ist freilich unterwegs. Von Politik und Gesetzgebung wird zunehmend erkannt, dass alle sozialen Sicherungssysteme und vor allem die klassischen Altersvorsorgesysteme zur Vermeidung der vorgenannten und weiteren Defizite allfälliger Entbürokratisierung, Deregulierung und Privatisierung bedürfen. Die gesamte Architektur der sozialen Sicherung und mit ihr die der Vorsorge verlangen angesichts der an sie gewendeten Modernisierungsanforderungen am Beginn des 21. Jahrhunderts nach umfassender Erneuerung. 43

Diese Zukunft hat schon begonnen. Die sog. Agenda 2010 der Bundesregierung vom 14. März 2003 markierte den Beginn des programmatischen Umbaus der sozialen Sicherungssysteme in Deutschland. ${ }^{44}$ Seine erklärten Ziele liegen im Rückschnitt des Wohlfahrtsstaates sowie in der Stärkung individueller Eigenverantwortung auch für die Vorsorge. Das Programm will ferner die Wirtschaft von zu hohen Arbeitskosten entlasten und zugleich die Haushaltssanierung fördern.

Ausfluss dieser Konzeption sind zumal die jüngsten Modernisierungsschritte in der Kranken- und Rentenversicherung. ${ }^{45}$ Zwar ist fraglich, ob die voraufgehend skizzierten Vorsorgedefizite überhaupt vermeidbar sind. Denn sie erweisen sich als das Resultat vorgegebener Ungleichheit und ein Systemwechsel macht sie nicht ohne weiteres vermeidbar. Doch gehen die Modernisierungsansätze immerhin in die richtige Richtung, wenngleich nicht wenige darunter die Abhängigkeit von der öffentlichen Sicherung und weniger die soziale Eigenvorsorge fördern. Verfehlt wird zugleich das Ziel, wie sich an der Debatte um die Altersteilzeit erkennen lässt, die finanzielle Krise des deutschen Vorsorgesystems zu beenden, weil die bisherigen Fehlanreize für den Umgang mit Sozialleistungen teilweise fortbestehen. So konterkariert in der Rentenversicherung eine Altersteilzeit als durchgängige Vergünstigung die jüngste Anhebung der Rentenaltersgrenze. Doch der Reihe nach.

43 Pitschas (Fußn. 2), VVDStRL Bd. 64 (2005), S. 128 ff.; Zacher, Der Sozialstaat an der Wende zum 21. Jahrhundert, VSSR 2000, S. $185 \mathrm{ff}$.

44 Agenda 2010 - Innovation und Wachstum. Regierungserklärung von Bundeskanzler Schröder am 14. März 2003 vor dem Deutschen Bundestag.

45 Für die Krankenversicherung vgl. Orlowski, Gesundheitsfonds und Dachverband - wo steht die Krankenversicherung in fünf Jahren?, in: Voit (Hrsg.), Gesundheitsreform 2007, Rechtliche Bewertung und Handlungsoptionen, Baden-Baden 2008, S. 66 ff.; zur Rentenversicherung s. Ruland (Fußn. 17), SGb 2008, passim. 


\section{Die Gesundheitsreform 2007 als „,Systemwandel“}

In der gesetzlichen Krankenversicherung (GKV) ist nach langwierigen und spannungsreichen Auseinandersetzungen innerhalb und außerhalb des Deutschen Bundestages nunmehr und zu dem weitaus größeren Teil das vielgescholtene „Gesetz zur Stärkung des Wettbewerbs in der gesetzlichen Krankenversicherung“ (GKV-Wettbewerbsstärkungsgesetz - GKV-WSG) am 1. April 2007 in Kraft getreten. ${ }^{46}$ Im Verlauf der Umsetzung dieses Reformwerks dürfte die vorherrschende Grundfrage zu einer Antwort finden, ob es tatsächlich ein zukunftsfestes und leistungsfähiges solidarisches Gesundheitssystem begründen wird, dass durch seine stärker dem Wettbewerbsdenken geöffneten Strukturen die Qualität und Wirtschaftlichkeit der Gesundheitsversorgung in Deutschland zu verbessern geeignet ist.

Die Zweifel hieran sind ebenso verbreitet wie das Vertrauen in das erfolgreiche Wirken des Gesetzgebers betont wird. Die einzelnen Regelungsgesamtheiten des Modernisierungsgesetzes sind an dieser Stelle nicht gesondert hervorzuheben. $\mathrm{Zu}$ betonen ist allerdings, dass die mit dem GKV-WSG intendierte „Gesundheitsreform 2007“ stets in Verbindung mit dem am 1. Januar 2004 in Kraft getretenen „Gesetz zur Modernisierung der gesetzlichen Krankenversicherung" sowie mit dem zum 1. Januar 2007 in Kraft getretenen „Gesetz zur Änderung des Vertragsarztrechts und anderer Gesetze“ zu sehen ist. Erst zusammengenommen bilden diese drei Gesetzesvorhaben in ihrer Gesamtheit den maßgeblichen corpus der sog. Gesundheitsreform 2007.47

\section{Systemwandel in der gesetzlichen Krankenversicherung}

Der Gesetzgeber hat mit diesen Gesetzesvorhaben einen Modernisierungsprozess in Gang gesetzt, der im „,neuen sozialen Rechtsstaat“ den unvermeidbaren Systemwandel der GKV - eingebettet in den allgemeinen Strukturwandel der deutschen Sozialversicherung und in Verbindung mit einer Neupositionierung der privaten Krankenversicherung (PKV) - herbeiführen wird. Dabei geht es vor allem um die Modernisierung des Sozialstaats am Beginn des 21. Jahrhunderts aufgrund der vielfältigen Probleme für die soziale Vorsorge, Gleichheit und „Wohlstandsteilhabe“, die aus den weltweiten ökono-

46 Gesetz vom 26.03.2007 (BGBl. I 378); dazu Bitter, Das GKV-Wettbewerbsstärkungsgesetz (GKVWSG) im Überblick, GesR 2007, S. 152 ff.

47 Pitschas, Die Gesundheitsreform 2007 - Verfassungskonformer Einstieg in den Systemwechsel der GKV, GesR 2008, S. 64 ff. m. Nachw. zu den Fundstellen der im Text zit. Gesetze in Fußn. 8 des Beitrags. Als gesetzlicher Regelungsauftrag aus der Gesundheitsreform 2007 entstand zwischenzeitlich das „Gesetz zur Weiterentwicklung der Organisationsstrukturen in der gesetzlichen Krankenversicherung“ (GKV-OrgWG) v. 15.12.2008 (BGB1. I S. 2426); dazu Füsser, GKV-OrgWG - Gesetz zur Weiterentwicklung der Organisationsstrukturen in der gesetzlichen Krankenversicherung Inhalte und Motive, SGb 2009, S. 126 ff. 
mischen, demografischen und wertebezogenen Verwerfungen in allen Sicherungssystemen resultieren.

Indessen sollte klar sein, dass der Gesetzgeber auf dem Boden des deutschen Grundgesetzes und angesichts des Vorrangs des europäischen Verfassungs- und Sozialrechts nicht schlagartig ein neues Gesundheitssystem installieren kann. Der verfassungsrechtlich gewährleistete und durch das Gemeinschaftsrecht unterlegte Sozialstaat darf die bereits ausgeführte staatliche Grundaufgabe nicht aus dem Auge verlieren, zur Sicherung der Menschwürde und freiheitlich-gleichheitlichen Existenz im Sozialstaat entsprechende solidarische und sozialgerechte Sicherungssysteme für die Hauptrisiken der Krankheit aufzubauen bzw. zu gewährleisten. Diese „Grundversorgung“ seiner Bürger ist staatliche Kernaufgabe und Gegenstand staatlicher Schutzpflicht. Bei deren Bewältigung liegt es aber nahe und ist legitim, auch die Trennlinie zwischen den sozialen Aktivitäten des staatlichen und des privaten Sektors neu zu ziehen, d. h. in der Gesundheitsversorgung auch das bisherige Nebeneinander von PKV und GKV neu zu ordnen. ${ }^{48}$

Einigkeit besteht in der Folge dessen jedenfalls darüber, dass die Antworten auf den sozialen Modernisierungsdruck stärker als zuvor den marktwirtschaftlichen Wechselbezug von Sozial- und Wirtschaftspolitik in Rechnung zu stellen haben. Hieraus resultiert die verfassungslegitime Forderung, die Realisierung des sozialstaatlichen Auftrags durch die Konkretisierung von Rechtsnormen im Sozialrecht auch an den Erträgen von Markt und Wettbewerb zu orientieren. Es gilt, auf diese Weise vermehrt die Wirtschaftlichkeit der Leistungserbringung einzufordern, ohne dadurch den Sozialstaat aus einer marktökonomischen Wurzel zu radizieren. Strukturell darf in der Gesellschaft des Grundgesetzes wegen des Verfassungsprinzips der „sozialen Gleichheit“ ein Mindest$\mathrm{ma}$ an sozialer Gerechtigkeit, an Solidarität und an sozialem Ausgleich in der Gesundheitsversorgung nicht unterschritten werden.

\section{Merkmale des Systemwandels und ,Wettbewerb“ als Steuerungskonzept}

Deshalb bleiben die Merkmale des „Systemwandels“ in der GKV in Erinnerung zu rufen. Den vorderen Platz gebührt dabei das neue Finanzierungsmodell für das GKVSystem, dass einen fundamentalen Wandel der gesundheitsbezogenen „Sozialversicherung" aus der Kombination einer Art Bürgerversicherung mit dem Gedanken der „Kopfpauschale“ einleitet. Es kommt zu einer Schwerpunktverlagerung weg von der bisherigen finanziellen Selbstregulierung der gesetzlichen Krankenkassen im Hinblick auf Finanzierung und Leistung und hin zur künftigen Funktion der Kassen als gemeinsamer Einkaufs- und Regulierungsagentur für Versorgungsleistungen - koordiniert durch den Spitzenverband Bund.

Dahinter scheint das anglo-amerikanische „HMO“-Modell auf. Durch gesetzgeberische „Regulierung“ auf der Einnahmenseite entsteht jedoch keine privatrechtsförmige,

48 Vgl. auch BVerfGE 103, 197 (216 ff.). 
sondern eine zentralstaatliche Gesundheitsagentur, genannt „Gesundheitsfonds“. Tendenziell läuft diese Strukturveränderung auf eine Grundsicherung in der Gesundheitsversorgung hinaus, die zugleich auf einer neuen, aber noch latenten Public Private Partnership der GKV mit der PKV beruht, die von den Richtlinien des Gemeinsamen Bundesausschusses flankiert wird. Der daraus resultierende Regulierungsdruck auf die Angebote der Leistungserbringer liegt auf der Hand. 49

Erwähnenswert, hier aber nicht weiter zu vertiefen sind daneben die Einführung eines „Basistarifs“ in der PKV und die parallel zum gegenwärtigen Strukturwandel des Gemeinsamen Bundesausschusses eintretende systemverändernde institutionelle Modernisierung der GKV auf der Grundlage der Errichtung des neuen Spitzenverbandes „Bund“. Verfolgt man die in dem Gesamtbild dieses Segments des Wandels erkennbaren Pfade näher, so scheinen die Weichen in Richtung auf die Entwicklung öffentlichrechtlicher und einheitlicher Krankenkassenkonzerne neben der PKV bei gleichzeitig umfassender Regulierung beider gestellt zu sein.

Ergänzend zu diesem Tableau tritt ein zweites Merkmal des Systemwandels in den Vordergrund: Die eingangs aufgeführten Gesetzeswerke lassen in ihrem Zusammenwirken ein spezifisches Bild der Steuerung des Beitrags- und Leistungsgeschehens durch „Wettbewerb" erkennen. ${ }^{50}$ Hervor leuchtet zunächst und einerseits ein durchgreifender Kassenwettbewerb, der durch den Abschluss der Entschuldung der Kassen zum 31.12.2008 sowie durch die Einführung des Insolvenzrechts in der GKV flankiert wird. ${ }^{51}$ Mir ist freilich zweifelhaft, ob von einer echten „Insolvenz“ der gesetzlichen Krankenkassen von Verfassungs wegen überhaupt die Rede sein darf und wen ggf. die Folgen treffen werden.

Auf der anderen Seite sieht sich der bislang schon gegebene vertragsgestützte Beitrags- und Leistungswettbewerb erheblich ausgeweitet und mit einer veränderten Steuerungsfunktion ausgestattet. Der Gesetzgeber setzt durch die Möglichkeit von Selektivverträgen und Arzneimittel-Rabattverträgen auf die Effizienzvermutung des Vertragswettbewerbs. Zudem sind es nicht nur die Einzelverträge, besonders im Bereich der Integrierten Versorgung, die im Trend liegen. Auch die Selbstverwaltungsträger der gesetzlichen Krankenkassen wie die Kassenärztliche Bundesvereinigung (KBV) sehen für sich als Partner des Kollektivvertragssystems neue Vertragsformen heranreifen, werden allerdings in dieser Erwartung auch teilweise enttäuscht, wie § 73 SGB V zeigt. Vertragswettbewerb wird jedenfalls als neuartige Steuerungsfunktion offenbar, so dass die kritische Analyse der Gesundheitsreform 2010 viel zu kurz greifen würde, wenn man sich ausschließlich den mit Selektivverträgen verbundenen wettbewerbsrechtlichen

49 Dazu s. am Beispiel des $\S 116$ b SGB V und seiner Umsetzung durch die Gesundheitsverwaltung Pitschas, Änderung der Versorgungsstrukturen durch Verflechtung von Leistungssektoren: Ambulante Krankenhausbehandlung nach § 116b SGB V, MedR 2008, 473 ff.

50 Näher insoweit die Beiträge in Pitschas (Hrsg.), Gesetzliche Krankenversicherung und Wettbewerb, Frankfurt a.M. 2008.

51 Gaßner, Neuregelung des Insolvenzrechts der Krankenkassen - Bewertung der Regelungen des GKV-WSG und des GKV-OrgWG aus Ländersicht, GesR 2009, S. 121 ff. 
Fragen der Vertragsgerechtigkeit, Monopolabwehr und des Kartellvergaberechts im Zusammenhang mit $§ 69$ SGB V widmen wollte. 52

\section{3. ,,Status-Passage“ für freie Berufe und gelenkter Vertragswettbewerb}

Wendet man sich statt dessen dem Vertragswettbewerb als Steuerungskonzept zu, dann geht es zum einen um die Vertragskonzeption sozialstaatlicher Modernisierungspolitik in der Vorsorge, zum anderen um die nunmehr bei der vertraglichen Konkretisierung von Rechtsnormen im Sozialrecht bestehende Typologie von Vertragsformen. In Verbindung damit zeigt sich sehr deutlich der gegenüber den ärztlichen Leistungserbringern eingeschlagene Weg, der vom Status des Inhabers eines freien Berufs weg und zum Vertrags-Dienstleister hinführt („Status-Passage“).

Diese Entwicklung ist Teil der sozialstaatlichen Modernisierung des Gesundheitswesens wie der Sozialversicherung insgesamt. Nicht nur in den Sonderverträgen nach $\S \S 73$ b, 73 c und 140 a SGB V ist insoweit ein Vertragskonzept aktivierender Wettbewerbspolitik im Gesundheitssektor angelegt. Der Modernisierung des Sozialstaates insgesamt ist der Übergang von gesetzlich begründeten Rechten und Pflichten, die einen subjektiv-rechtlichen Anspruch auf Leistungserbringung begründen, zum vertraglichen Zusammenwirken eigen. ${ }^{53}$ In dessen Verlauf wird fixiert, was die Vertragspartner als richtig akzeptiert haben und ihren Interessen gemäß festsetzen. Ein Anspruch auf Vertragsschluss, etwa nach $\S 73 \mathrm{c}$ Abs. 1 SGB V besteht allerdings nicht. Gegenstand der Verträge können auch gesamtheitliche Versorgungsaufträge sein, die sowohl die versichertenbezogene gesamte ambulante ärztliche Versorgung als auch einzelne Bereiche dieser umfassen.

Neben dem Abschluss von Einzel- bzw. Strukturverträgen mit Leistungserbringern, in denen auch die nach dem Gesetz vorzuhaltenden ärztlichen Leistungen konkretisiert werden, ist überdies an die Ausweitung des Kollektivvertragssystems in den PKVVergütungsbereich gem. § 75 Abs. 3 b SGB V zu erinnern. Denn das Wettbewerbsstärkungsgesetz fügt dem Gesetz eine Versicherungspflicht für alle Arbeitnehmer in der GKV ein und verbindet damit die Möglichkeit, auch den Beitritt zur PKV zu wählen. Diese wird verpflichtet, für alle Nicht-Versicherten zum 1.1.2009 ein sog. „Basistarif“ anzubieten (§ 257 Abs. 2 a SGB V). Insofern wird Kontrahierungszwang angeordnet. ${ }^{54}$

52 Skeptisch daher zur Reichweite der Wettbewerbsmechanismen Gaßner (Fußn. 51), der von einem „Wettbewerbseuphemismus“" spricht (S. 122 1. Sp.).

53 Zum „Vertragskonzept aktivierender Sozialpolitik“ prononciert Ebsen, Der Arbeitslose als Sozialbürger und Klient - Der Betroffene im Konzept des aktivierenden Sozialstaates, in: v. Wulffen/Krasney (Hrsg.), Festschrift 50 Jahre BSG, Köln 2004, S. 725 (736 ff.).

54 Näher dazu Pitschas, in: Kluth/Müller/Peilert (Hrsg.), FS für R. Stober (Fußn. 34), S. 306 ff. 


\section{Divergenzen zur herkömmlichen vertraglichen Konkretisierung von}

\section{Rechtsnormen}

Der Einsatz von Verträgen im Sozialrecht zur Konkretisierung der durch den Gesetzgeber erlassenen Rechtsnormen ist nicht ungewöhnlich. Das Verwaltungsverfahrensrecht und diesem entsprechend das SGB X haben den öffentlich-rechtlichen Vertrag als zweite wichtige Handlungsform neben dem Verwaltungsakt (Bescheid) gesetzlich geregelt. Dahinter steht der Gedanke, dass der moderne Sozialstaat, wie schon erläutert, auch auf eigenverantwortliches Handeln und die hierfür erforderliche (Vertrags-) Autonomie des Einzelnen setzt. 55

Gleichwohl kommt in der Rechtspraxis dem öffentlich-rechtlichen Vertrag trotz weitgehender Ausgestaltung der Vorsorge als Leistungsrecht im Verhältnis von Sozialleistungsträgern untereinander und zu den Leistungsempfängern bisher keine wesentliche Bedeutung zu. Eine Ausnahme bilden die kollektivrechtlichen Verträge, die vor allem im Kassenarzt- bzw. Vertragsarztrecht, des Weiteren aber auch und z.B. im Krankenhausrecht und im Recht der sozialen Pflegeversicherung ihre Heimstatt finden. Eine Sonderform bilden die Gesamtverträge auf Bundesebene, bei denen es sich um sog. Normsetzungsverträge handelt. ${ }^{56}$ Deren allgemeinen Inhalt vereinbaren die Selbstverwaltungsträger auf der Seite der Leistungserbringer mit den Spitzenverbänden der Krankenkassen in sog. Bundesmantelverträgen. Der Inhalt der Bundesmantelverträge ist Bestandteil der Gesamtverträge.

Auf diese Weise kommt es zwar schon bisher zur vertraglichen Konkretisierung der gesetzgeberischen Anordnungen im SGB V. Doch lässt der Gesetzgeber nunmehr im Rahmen der Gesundheitsreform 2007 auch Sonderverträge an den Kassenärztlichen Vereinigungen vorbei zu. Dadurch kommt es zu einer Funktionsänderung vertraglicher Normkonkretisierung, weil nicht mehr ausschließlich in kollektiver Form, sondern in Nutzung privatautonomer Vertragsabschlusskompetenz der einzelne Leistungserbringer zu einer Vereinbarung mit einer gesetzlichen Krankenkasse selbst regional finden kann. Damit einher gehen ein Funktionswandel der Bundesmantelverträge und ein interner Steuerungsverlust der Selbstverwaltungsgremien auf Leistungserbringerseite.

Der Funktionswandel vertraglicher Normkonkretisierung ist im Übrigen nicht so unproblematisch, wie es scheint. Zwar spricht viel dafür, dass vertragliches Zusammenwirken für das ausbedungene Ergebnis eher aktive Unterstützung auf dem Weg dorthin von allen Vertragsparteien mobilisieren kann. Gleichwohl dürften sich damit auch Nachteile für den einzelnen Leistungserbringer in der Krankenversicherung einstellen. Denn das Vertragskonzept setzt zwar Rechtsgleichheit in dem Sinne voraus, dass man nicht verpflichtet ist, den Vertrag zu den Konditionen der anderen Vertragsseite zu

55 Pitschas, Das sozialrechtliche Verwaltungsverfahren im ,aktivierenden“ Sozialstaat. Verfahrensrechtliche Konsequenzen der staatlichen Verantwortungspartnerschaft mit der Bürgergesellschaft, in: v. Wulffen/Krasney (Hrsg.), FS 50 Jahre BSG (Fußn. 53), S. 765 (766 f., 772 ff.).

56 Engelmann, in: v. Wulffen, SGB X, Kommentar, 6. Aufl. München 2008, § 53 Rdnr. 3, 48 ff. 
schließen. Doch liegen die Dinge anders, wenn man - wie für den vertragsärztlichen Sektor beispielsweise - in der GKV eine gewisse Asymmetrie der Vertragsbeziehungen feststellen kann. Gleiches gilt im Arbeitsförderungsrecht nach dem SGB III. Denn im Verhältnis zu den mächtigen Vertragspartnern auf der Kassen- bzw. Agenturseite fehlt es an einer entsprechenden Vertragsparität. Das Vertragskonzept vermag aber die ihm zugemessene Funktion nur zu erfüllen und den Verlust des durch die Kollektivverträge an sich gewährleisteten Status durch Vertragssicherheit zu kompensieren, wenn alle Vertragspartner - auch bei Einzelverträgen - „mit gleich langen Spießen“ handeln würden. Die hilfsweise Bereitstellung von Schiedsämtern im Fall von Normsetzungsverträgen bietet demgegenüber keine wirkliche Kompensation.

\section{Auf dem Weg zur Vereinheitlichung von öffentlicher und privater}

\section{Krankenversicherung}

Der gelenkte Vertragswettbewerb, der sich nach alledem als ein durchaus problematisches Steuerungskonzept in der GKV darstellt, will offenkundig die Gesundheitsvorsorge durch Privatversicherung mit der Zuweisung von Wettbewerbsfunktionen an die GKV unter gleichzeitiger Einschränkung der Unternehmensfreiheit auf der PKV-Seite noch stärker als bisher ,solidarisch“" ausgestalten.

Soll man diese Entwicklung beurteilen, kommt es auf den Standpunkt des Betrachters bei der verfassungsrechtlichen Einschätzung der Reichweite staatlicher Verantwortung für soziale Vorsorge an. Die Erweiterung der Wahlmöglichkeiten der Versicherten (Wahltarife), die Portabilität der Altersrückstellungen in der PKV sowie die erweiterten Vertragsmöglichkeiten der GKV könnten auch zum verstärkten Wettbewerb unter Leistungserbringern und im Arzneimittelbereich führen. Alle Anzeichen sprechen dafür. Dies aber bedeutet, dass neue Regulierungen mit Vertragsanreizen so verknüpft werden, dass als Ergebnis der Anreizregulierung nicht etwa die patientenzentrierte oder versichertenorientierte Eigenvorsorge für die Gesundheit angestrebt wird, sondern die Fremdbestimmung durch zentralstaatliche oder mächtige privatwirtschaftliche Institutionen. Letztlich befindet sich damit die Gesetzgebungspolitik auf dem Weg zu einer Vereinheitlichung von öffentlicher und privater Krankenversicherung - einem Ziel, das durchaus gemeinschaftsrechtlichen Vorstellungen entspricht -, aber gleichzeitig auf dem Weg zur Patienten- und Verbraucherferne. 57

\section{Geltung des Wettbewerbs- und Kartellvergaberechts}

Ob der durch die Gesundheitsreform 2007 geöffnete Markt für Vertragsabschlüsse, Wahltarife und Zusatzversicherungen, der bisher allein den privaten Krankenversiche-

57 Vgl. das Ergebnis der dokumentierten Podiumsdiskussion „Patientenschutz“ in Voit (Hrsg.), Gesundheitsreform 2007 (Fußn. 45), S. 45 ff. 
rungen vorbehalten war, tatsächlich zu mehr sozialer Freiheit auf der Seite der Versicherten und Verbraucher führt, ist also zweifelhaft. In dieser Skepsis bestärkt den Rechtsanwender die aktuelle Regelung des $\S 69$ SGB V, wonach das Wettbewerbsrecht einschließlich des Kartellvergaberechts im Sozialsektor nur beschränkte und „entsprechende" Anwendung finden soll. Hierzu hat unter dem Titel und mit der klaren Aussage „Keine Geltung des Kartellvergaberechts für Selektivverträge der Krankenkassen mit Leistungserbringern" der frühere Vorsitzende des 6. Senats des BSG selbst vor kurzem ausführlich Stellung bezogen. ${ }^{58}$ Das BSG hat sich hierzu in der Sache noch nicht abschließend geäußert, sondern lediglich aufgrund einer Rechtswegbeschwerde den Rechtsweg zu den Sozialgerichten für zulässig erklärt. Der dem vorausgegangene Beschluss des LSG Baden-Württemberg vom 6. Februar $2008^{59}$ spricht sich zwar gegen die Anwendung des Kartellvergaberechts aus, lässt aber eine (nur unklar begründete) öffentlich-rechtliche Wettbewerbskontrolle durch Verpflichtung der Vergabe auf Grundsätze der Fairness bzw. der Transparenz im Vergabehandeln zu. In der Folge dessen können Rabattverträge zwischen gesetzlichen Krankenkassen und Pharmaunternehmen derzeit nicht zum Zuge kommen.

Ich habe demgegenüber keinen Zweifel daran, dass die Anwendung des Vergaberechts der Rechtslage in der EU entspricht. Zwar stellt diese dem deutschen Gesetzgeber frei, innerhalb des Sektors der Daseinsvorsorge überwiegend im nationalen Alleingang Regelungen zu treffen. Gleichwohl ist der vorgegebene Rahmen des europäischen Wettbewerbsrechts zu beachten, wonach die Sozialversicherung der Wettbewerbskontrolle unterliegt, wenn ihr Aufgaben zugewiesen werden, die auch von privaten Unternehmen auf freien Märkten erbracht werden können. Mir scheint, die Vertragskonzeption und die Öffnung der Gesundheitsmärkte für Wahltarife und Zusatzversicherungen im Angebot durch die GKV bilden einen solchen Fall. Denn die GKV ist als eine wirtschaftliche Einheit und infolge ihrer Wirtschaftskraft als ein marktbeherrschendes Unternehmen anzusehen, dem Art. $81 \mathrm{f}$. EGV den Verdrängungswettbewerb von Wettbewerbern untersagt. Deshalb und ferner ist nicht nur ein eventueller Marktmachtmissbrauch durch die GKV vor den Sozialgerichten der Kontrolle unterworfen, sondern auch die Vergabepraxis. ${ }^{60}$ Wer diese Kontrolle näherhin wahrnimmt, Sozialgerichtsbarkeit oder Zivilgerichtsbarkeit, hat der Gesetzgeber zugunsten der ersteren entschieden - eine Antwort des politischen Machtspiels, die an dieser Stelle nicht näher interessieren soll.

58 Engelmann, Keine Geltung des Kartellvergaberechts für Selektivverträge der Krankenkassen mit Leistungserbringern, SGb 2008, S. $133 \mathrm{ff}$.

59 BSG, NZS 2009, S. 38; LSG Bad.-Württ., NZS 2008, S. 384.

60 Dies deutet das BSG, ebda. (Fußn. 59) in Rdnr. 54 seiner Entscheidung an. 


\section{Neue Architektur der gesetzlichen Rentenversicherung}

\section{Die gesetzliche Rentenversicherung als „,Grundsystem“ der Altersvorsorge}

Ebenso wie die anderen Teil-Systeme der Sozialversicherung unterliegt auch die gesetzliche Rentenversicherung (RV) in ihrer mehr als hundertjährigen Entwicklungsgeschichte seit 1881 einer prozess- und strukturbezogenen Veränderungsdynamik. ${ }^{61}$ Soziale Sicherung im Alter gegen den Verlust des Arbeitseinkommens oder wegen Invalidität ist jedoch dem Grundgesetz selbstverständlich und von Verfassungs wegen als gesetzliche Rentenversicherung einem gesondert dafür geschaffenen öffentlich-rechtlichen Sicherungssystem anvertraut. Das BVerfG hat es ,als das Grundsystem sozialer Sicherung" bezeichnet und als dessen Eckpfeiler das Sozialstaatsprinzip ausgewiesen. ${ }^{62}$ Dieses gibt dem Gesetzgeber unter Beachtung seiner Verankerung im „sozialen Bundesstaat" auf, die RV entsprechend auszugestalten, ohne jedoch Grundzüge der Konkretisierung oder Einzelheiten zu nennen. Es gibt deshalb auch keine sozialstaatliche Systemgarantie.

Gleichwohl ist nach Maßgabe des Verfassungsprinzips Sicherheit im Sozialstaat von einer Mindestsicherungsfunktion staatlicher RV auszugehen. Jedenfalls hat nach der Vorstellung des Grundgesetzes in jenen Fällen der Staat einzuspringen, in denen Bürger nicht selbst die im Alter ausreichende soziale Sicherheit erreichen können. Allerdings setzt dieses Verständnis der Mindestsicherungsfunktion eine generalisierte Vorsorgefähigkeit und strukturell die Möglichkeit zur eigenständigen Absicherung des Altersrisikos, z.B. durch berufliche Erwerbstätigkeit voraus.

Die Rechtsprechung des BVerfG liegt auf dieser Linie: Die Sicherungsfunktion der RV soll gewährleistet sein. In diesem Sinne versteht sich die Verfassung als ein struktureller Gesamtrahmen für jedwede Alterssicherungskonzeption. Das (soziale) Sicherheitsprinzip will dem beitragszahlenden Sozialversicherten eine Leistung garantieren, die zumindest den Einkommensersatz sichert - wie auch immer dieser unter Einbezug von Ergänzungssystemen durch den Gesetzgeber konkretisiert wird.

Damit sieht sich die gesetzliche Rente bisher als eine Lebensstandardsicherung konzipiert, die durch die Einkommensbezogenheit der Beiträge und die Beitragsbezogenheit der Leistungen gewährleistet wird. Ihre Lohnersatzfunktion wird deshalb auch nur erfüllt, wenn die staatliche Rente zukünftig das Niveau der Sozialhilfe oder anderer Basissysteme der sozialen Sicherung überschreitet und sich in ihrer Form hinreichend von einer Grundsicherung unterscheidet. Sie darf also nicht etwa von Bedürftigkeitsprüfungen abhängig gemacht werden. ${ }^{63}$

61 Döring, Grundlinien der langfristigen Systementwicklung der gesetzlichen Rentenversicherung Personenkreis, Rentenformel, Finanzierung -, in: Fisch/Haerendel (Hrsg.), Geschichte und Gegenwart der Rentenversicherung in Deutschland, Berlin 2000, S. $169 \mathrm{ff}$.

62 BVerfGE 98, 1 (16); 100, 1 (39).

63 Ruland (Fußn. 17), SGb 2008, S. 574 f. 


\section{Grundsicherung durch steuerfinanzierte Sockelrente?}

Nicht unbedingt ist aber von Verfassungs wegen die Beitragsfinanzierung staatlicher Alterssicherung vorgegeben. Auch garantiert die Konnexität von Beitrag und Leistung keine strikte „Individualäquivalenz“. Die Zugehörigkeit zum System der Altersvorsorge in Deutschland vermittelt vielmehr nur eine „Anwartschaft“ darauf, später im Wege der umlagefinanzierten Rente die versprochene Leistung zu erhalten. Es handelt sich um Teilhabe an den vor Eintritt des Versicherungsfalls im Wege des Umlageverfahrens angesammelten Finanzmassen (,Teilhabeäquivalenz").

Vor diesem Hintergrund erscheint künftig selbst der Übergang zu einer Alterssicherung durch Staatsbürgerversicherung, die das Alterseinkommen von lohnbezogenen Beiträgen abzukoppeln versuchen und eine einheitliche „Pauschalprämie“ für eine Grundsicherung vorsehen würde, rechtlich zulässig. ${ }^{64}$ Zusatzsysteme auf der Grundlage einer Altersvorsorge durch Privatversicherung könnten dann - wie bereits derzeit die betriebliche Altersversorgung - als freiwillig ergänzende Vorsorge i. S. eines Mehrsäulen-Modells zur Alterssicherung hinzutreten, obschon solche Sonderformen aus Gründen der Effektivität und Effizienz auskömmlicher Alterssicherung gering zu halten wären. $\mathrm{Zu}$ bedenken ist dabei überdies und einerseits die möglicherweise eintretende Gleichheitsdifferenzierung, andererseits der in den letzten Jahren stetig zu einer „Bürgersicherungsleistung“ angewachsene Bundeszuschuss. In der jüngsten Diskussion wird denn auch empfohlen, eine steuerfinanzierte Sockelrente zu prüfen, auf die andere Alterseinkünfte und Vermögen der Ruheständler nicht angerechnet werden dürfen. Dies freilich ist inkonsequent, wenn man zugleich auf Beitragsfinanzierung verzichtet. 65

\section{Zukunftsdefizite der gesetzlichen Rentenversicherung}

Im Ergebnis ist ein bedingungsloses Grundeinkommen von der Sozialhilfe nur schwer abzuschichten. Soweit es von verschiedenen Seiten mit unterschiedlichen Konzeptionen als eine Möglichkeit diskutiert wird, Finanzierung und Ansprüche sozialer Alterssicherung auf eine neue Grundlage zu stellen, bedarf es jedenfalls eines umfassenderen Systemwechsels, der über das Vorsorgeverhältnis hinausreicht.

Der vorgestellten Architektur der gesetzlichen RV mit ihren Modernisierungsansätzen drohen allerdings immer schneller die Fundamente wegzubrechen. Im Vordergrund steht dabei und einerseits die Finanzierungsproblematik aufgrund der demografischen Entwicklung. So wird der Anteil der Erwerbsbevölkerung in den kommenden Jahren deutlich zurückgehen, wodurch ein immer größeres Missverhältnis zwischen dem erwerbstätigen Teil der Bevölkerung und den Rentenversicherten eintreten wird. Dement-

64 Vgl. auch Lenze, Staatsbürgerversicherung und Verfassung, Tübingen 2005, passim.

65 Preis (Fußn. 31), S. B 66 f. 
sprechend hat in der RV der sog. Generationenvertrag zur Begründung künftiger sozialstaatlicher Alterssicherung ausgedient.

Die Umlage finanzierter Alterssicherung gerät aber nicht nur dadurch zunehmend unter finanzwirtschaftlichen Druck. Probleme bereitet insofern auch und andererseits, dass die Grenzen der Steigerung von Produktivität und Kapitaleinsatz auf dem Arbeitsmarkt fast erreicht sind. Der Produktionsfaktor Arbeit darf sich aber nicht zu einer „Wachstumsbremse“ entwickeln, weil andernfalls das bisherige Wohlstandsniveau mit seinem Anteil an der Sicherstellung auskömmlicher Renten nicht beibehalten werden kann. Mit Erreichen einer Regelaltersgrenze darf deshalb in der gesetzlichen Rentenversicherung nicht argumentiert werden. Absolute Altersgrenzen für den Ausstieg aus dem Berufsleben sollten entfallen. Dies ist rechtsvergleichend eine Lösung, wie sie etwa in den Niederlanden unlängst eingeführt worden ist. 66

Mit Sorge ist darüber hinaus die absehbare Entwicklung zu einem gespaltenen „Rentenniveau“ zu beobachten. Denn diejenigen, die nach dem Jahr 2011 in Rente gehen, werden deutlich geringere Renten beziehen als diejenigen, die schon vor diesem Zeitpunkt Rente erhielten. Ein darauf abzielender „Ausgleichsfaktor“ ist indes verfassungsrechtlich ebenso problematisch wie die geplante Absenkung des Rentenniveaus bis zu 46\% im Jahr 2020. Damit aber ist vor Art. 14 GG die Frage nach den verfassungsrechtlichen Folgen einer Negativrendite aus dem Umlaufverfahren aufgeworfen. ${ }^{67}$

\section{Konzeptionelle Ansätze zu einer ,neuen“ Altersvorsorge:}

\section{Die Mehrsäulen-Strategie}

In der Diskussion über diese Problemkreise ist von ,grundstürzenden Veränderungen“ die Rede, denen sich die Sozialstaatlichkeit ausgesetzt sehe. Doch vermag als Antwort auf die Zukunftsdefizite nur ein behutsamer Systemwandel in der gesetzlichen Altersvorsorge in Betracht kommen. 68 Sein Leitbild ist die Stärkung individueller Eigenvorsorgeverantwortung im Rahmen des subsidiären Sozialstaates. Die von diesem als „Steuerstaat“ mittlerweile ausgehende Förderung des Aufbaus eines Systems der privaten, kapitalgedeckten Alterssicherung und die Stärkung der betrieblichen Altersvorsorge entsprechen diesem Leitbild.

Mit der Hinwendung der gesetzlichen RV in den letzten Jahren zu einem neuen Konzept ihrer Finanzierung und Reichweite haben sich bereits wesentliche Elemente der

66 Vgl. ferner $O E C D$, Alterung und Beschäftigungspolitik, 2006, S. 33.

$67 \mathrm{Zu}$ dieser Diskussion s. etwa Papier, Alterssicherung und Eigentumsschutz, in: Isensee/Lecheler, Freiheit und Eigentum, Festschrift für Walter Leisner zum 70. Geburtstag, Berlin 1999, S. 721 (741); Pitschas, Verfassungsvoraussetzungen für die Entstaatlichung der gesetzlichen Rentenversicherung in Deutschland, in: Becker/Kaufmann/v. Maydell/Schmähl/Zacher (Hrsg.), FS für F. Ruland (Fußn. 30), S. 99 (106); Wenner, Rentenniveau und Grundgesetz, in: v. Wulffen/Krasney (Hrsg.), FS 50 Jahre BSG (Fußn. 53), S. 625 (633 ff.).

68 Papier, in: v. Maydell/Ruland/Becker (Hrsg.), Sozialrechtshandbuch (Fußn. 8), Rdnr. 94 („Systemwahrungsgebot“); Pitschas (Fußn. 2), VVDStRL Bd. 64 (2005), S. 136 („Kontinuitätsgrundsatz“). 
zukünftigen Architektur einer „gemischten Alterssicherung“ herausgeschält. Diese vertraut einem kooperativen Versicherungsmodell, das dem Gedanken der individuellen Eigenvorsorge bei staatlicher Förderung durch den Gesetzgeber gebührend Raum gibt. Ferner wird durch teilweise Kapitaldeckung der Alterssicherung der bemängelte $\mathrm{Zu}$ sammenhang zwischen Erwerbseinkommen und Rentenversicherungsbeiträgen zugunsten alternativer Finanzierungsweisen aufgelöst. Schließlich wird die Ausgabenseite der gesetzlichen RV entlastet.

Verantwortlich dafür zeichnen konzeptionelle Ansätze zu einer ,neuen Altersvorsorge“, die sich insgesamt als Mehrsäulen-Modell entfaltet. Neben die „erste“ Säule einer Basis- bzw. Grundsicherung durch die gesetzliche Rente wird die „Zweite“ Säule der betrieblichen Versorgung und die weitere Säule der privaten Altersvorsorge gesetzt. Der zusätzlich in letzter Zeit unterbreitete Vorschlag, als vierte Säule private Vorsorge durch Zusatzverdienste bis in das hohe Alter hinein zu ermöglichen ${ }^{69}$, überzeugt dagegen nicht. Er enthält verdeckt die Schmälerung des Alterseinkommens: Indem herkömmlicherweise und ausgehend von der Lohnersatzfunktion der Gesamtbezug von Altersvorsorgeleistungen einem Kumulierungsverbot unterworfen wird, tragen solche Zusatzverdienste dazu bei, den staatsseitigen Anteil an der Alterssicherung immer stärker schrumpfen zu lassen.

Richtig dagegen ist, dass vor diesem Hintergrund das Umlageverfahren als eine Art „Notsicherung“ in heiklen Zeiten bestehen bleiben sollte. 70 Problematisch dagegen scheint, dass jedenfalls das Renteneintrittsalter über das 67. Lebensjahr hinaus zu flexibilisieren wäre. Hinweise auf einen früheren Rentenabruf nach Wahl klingen gut, sind aber für den Betroffenen nichts anderes als eine verdeckte Rentenkürzung. Ob daran der Altersvorsorgevertrag (,Rürup-Rente“) etwas zu ändern vermag, ist fraglich.

\section{Verfassungsrechtliche Grenzziehungen für die Modernisierung des Rentensystems}

Das Grundgesetz formuliert kein elaboriertes Konzept für die Privatisierung der Altersvorsorge. Doch lassen sich im Richtsteig von individueller Verantwortung für die eigene Alterssicherung, den Rahmenbedingungen langfristiger Umorientierung der gesetzlichen RV auf ein Kapitaldeckungssystem und dem Optionenermessen des Gesetzgebers eine Reihe zentraler Verfassungsvoraussetzungen aufzeigen, die den Pfad zu einem künftigen Rentenkonzept weisen. ${ }^{71}$

Dabei steht im Mittelpunkt und einerseits Art. 14 Abs. 1 in Verbindung mit Art. 2 Abs. 1 GG. Freilich hat sich das Eigentumsgrundrecht bislang kaum jemals als Hindernis einer Modernisierung der RV erwiesen. Der prozedural verstandene Sozialstaat ge-

69 Preis (Fußn. 31), S. B $111 \mathrm{f}$.

$70 \operatorname{Krupp}$ (Fußn. 39), S. 183 ff., 185 (,,vernünftige Mischung“).

71 Vgl. Papier, in: Isensee/Lecheler, FS für W. Leisner (Fußn. 67), S. 721 zur Maßgabe des Art. 14 GG; Pitschas, in: Becker/Kaufmann/v. Maydell/Schmähl/Zacher (Hrsg.), FS für F. Ruland (Fußn. 67), bes. S. $112 \mathrm{ff}$. 
währt vielmehr sowie ungeachtet des der Eigentumsgarantie erfließenden Vertrauensschutzes den notwendigen gesetzgeberischen Entscheidungsspielraum bei Systemfragen. Darüber hinaus gibt es für die neue Architektur der RV kein geschlossenes Verfassungskonzept. Immerhin würde aber die Eigentumsgarantie als Schutznorm privatsozialer Sicherung gegenüber dem Rentengesetzgeber eine stärker effektive Direktive entfalten. Ihr entspringt auch der Auftrag an den Staat, i. S. einer Schutzpflicht erkennbare Gefährdungspotentiale privater Altersvorsorge gegenüber Dritten abzuwehren. Art. 14 GG leistet insofern - wie auch Art. 12 Abs. 1 GG bei der Sicherung des „Alterslohns“72 - einen Beitrag zur künftigen Regulierung der privaten Alterssicherung.

Dagegen erfüllt die Eigentumsgarantie keine Renditeerwartungen der Beitragszahler in der gesetzlichen RV.

Insofern schließlich das Verfassungsprinzip „Sicherheit“ über das dem Grundrecht aus Art. 2 Abs. 1 GG erfließenden Vertrauensschutz und die rechtsstaatliche „Rechtssicherheit" hinaus ${ }^{73}$ das Vertrauen in die Altersvorsorge schützt, werden jedenfalls einer mehr „privaten“ als „öffentlichen“ Alterssicherung Grenzen gezogen. Nicht zuletzt gebietet das Gleichbehandlungsgebot des Art. 3 Abs. 1 GG einen hinreichenden Abstand künftiger Altersvorsorge in der Niveausicherung gegenüber der Sozialhilfe bzw. allfälliger Mindestsicherung.

\section{E. Art und Maß der zukünftigen Vorsorge als „Neue soziale Frage“}

\section{Vorsorge durch „, Markt und Wettbewerb“-oder:}

Wer kann sich künftig welche Sicherung leisten?

Die skizzierten Gleichheitsdefizite der überkommenen Sozialversicherung wie überhaupt die innere Spannung zwischen staatlicher Vorsorgeverantwortung und gesellschaftlicher Vorsorgeautonomie verstärken sich im Zuge der Modernisierung aller sozialen Sicherungssysteme eher noch, als das sie zurückgehen würden. Auch für die soziale Vorsorge zeichnet sich, was den Zugang zur Erwerbsarbeit, das Leistungsniveau, die Bedarfsgerechtigkeit oder auch die gruppenhafte Typisierung von Sozialversicherungsleistungen und deren Finanzierung anbelangt, eine Konfrontation von Sicherheitserwartungen mit der neuen Wirklichkeit ab. Denn deren Gestaltung wird sowohl in der sozialen Rentenversicherung als auch in der gesetzlichen Krankenversicherung, zuneh-

72 Vgl. BVerfGE 120, 169 (177 f.; 179); in dieselbe Richtung zuvor bereits BVerfGE 105, 73 (127 f.); Pitschas, Der verfassungsrechtliche Schutz von Renten der Sozialversicherung AöR Bd. 107 (1982), S. 149 (151 f.); ders., Rentenanpassung oder Rentenreform? - Verfassungsprobleme um das 21. RAG, VSSR 1978, S. 357 (375 ff.).

73 Dazu Papier, in: v. Maydell/Ruland/Becker (Hrsg.), Sozialrechtshandbuch (Fußn. 8), Rdnr. 125, 130. 
mend ebenfalls in der Pflegeversicherung und ferner im Sektor der Arbeitsförderung den sich ausdehnenden privaten Angeboten zur Eigenvorsorge überantwortet; künftige soziale Sicherheit sieht sich damit den Gestaltungskräften von „Markt und Wettbewerb“ anheim gegeben.

Die Sicherung durch Sozialversicherung wird dadurch zu einem erheblichen Teil und womöglich dauerhaft - durch eine Sicherung seitens privater Vorsorgeeinrichtungen jenseits des für die staatliche Finanzierung typischen Umlageverfahrens ergänzt bzw. ersetzt. Der mit alledem gleichzeitig verbundene und jedenfalls teilweise Übergang zu einem Kapitaldeckungsverfahren geschieht allerdings in einem Zeitpunkt, in dem die „Krise des Sozialstaats“ das Vertrauen in der Bevölkerung auf die bisherige soziale Sicherung durch die Sozialversicherung erschüttert hat. Aufmerksam und mit Erbitterung werden die Reduktionen sozialer Leistungen in der Rentenversicherung, die deshalb auf die durchgehende Anwendung des sog. „Riester-Faktors“ auf Zeit verzichtet hat, oder auch in der Krankenversicherung durch Budgetrestriktionen und die Einführung zielgruppenbezogener Richtgrößen bei der Arzneimittelverschreibung registriert. Hinzu treten das Versagen der Finanzmärkte und die Instabilität der Kapitalanlagen.

Von daher stellt sich die Frage neu, wie umfangreich und kostspielig die öffentlichrechtliche Versorgung noch sein darf und wie viel private Vorsorge vom einzelnen ermöglicht werden kann. Im Zentrum der hierüber geführten Diskussionen steht denn auch mehr als je zuvor die Finanzierbarkeit der Renten oder auch einer umfassenden Gesundheitsversorgung. Wenn man so will, wird dadurch eine neue soziale Frage aufgeworfen, nämlich danach, wer sich künftig welche Gesundheitsversorgung noch leisten oder welche Alterssicherung erwerben kann.

\section{Probleme der Verweisung auf private Vorsorgeverantwortung}

Aus ökonomischer Perspektive erfährt die Überführung von Teilen der Vorsorge in private Verantwortung vielfältigen Zuspruch. Vermuteten Einschränkungen der gesellschaftlichen Solidarität wird die Überlegung entgegengestellt, dass mit der Selbstregulierung von Versorgungserwartungen und -ansprüchen bei gleichzeitiger Konzentration auf das Notwendige individueller Missbrauch und allfällige Fehlallokationen vermieden werden. ${ }^{74}$ Das wird zwar diejenigen weniger trösten, die heute bereits dem Unbill der gesellschaftlichen Armut ausgesetzt sind oder zu denjenigen Familien zählen, die staatliche Hilfe auf Dauer bitter notwendig haben. Rechtlich werden jedoch dieser Entwicklung keine Steine in den Weg gelegt, wie sich am Beispiel der Verweisung auf ergänzende private Vorsorge in der sozialen Rentenversicherung bzw. in der anhaltenden Ausgestaltung der Pflegeversicherung als Grundsicherung offenbart. Insofern, aber auch nur diesbezüglich zutreffend wird betont, dass unsere Verfassung zwar in der sozialen

74 Börsch-Supan, Über selbststabilisierende Rentensysteme, in: Becker/Kaufmann/v. Maydell/ Schmähl/Zacher (Hrsg.), FS für F. Ruland (Fußn. 30), S. 157 (163, 164 ff.). 
Sicherung eine grundlegende Staatsaufgabe sehe, nicht aber deren Ausführungswege und die überkommene Art, sie zu bewältigen, garantiere. Dementsprechend ist weder in der Rentenversicherung noch in der Krankenversicherung bislang erfolgreich gegen die Senkung der Leistungen oder für die Einführung einer sog. Bürgersozialversicherung in der gesetzlichen Krankenversicherung gestritten worden.

Gleichwohl zeigen die im Gewand anhaltender Diskussionen hierüber und über die Reichweite des Wettbewerbsrechts in Angelegenheiten der Sozialversicherung (vgl. $\S 69$ SGB V) bzw. die um die Geltung des Sozialvergaberechts geführte Auseinandersetzung über die Verweisung auf private Vorsorge einen fortbestehenden, offenkundigen Bedarf nach (verfassungs-)rechtlicher Klärung, ob und in welcher Weise eine Mindestpflicht des Sozialstaates besteht, entsprechend dem Verfassungsprinzip Sicherheit des Sozialstaates ein über das Existenzminimum hinausgehendes Versorgungsniveau sozialer Sicherheit durch staatliche Gewährleistung zu sichern. ${ }^{75}$ Mir scheint jedenfalls fraglich, ob die neuen und im Rahmen der Privatisierung sozialer Sicherung begründeten Formen der Vorsorge den Anforderungen der Grundrechte und des Sozialstaats genügen. Jedenfalls hat für die Krankenversicherung unlängst das sog. „Nikolaus-Urteil“ des BVerfG mit Recht bestätigt, dass nicht jedes Maß der sozialen Sicherung (hier: im Krankheitsfall) der politischen Entscheidung des Gesetzgebers überlassen bleiben darf. ${ }^{76}$ Das Ergebnis ist ein sozialrechtliches Untermaßverbot.

Verlängert man die damit eingeschlagene Linie der Argumentation, so sollte der Staat der sozialen Sicherheit jedenfalls nicht auf die Pflicht und die bloße Gestaltung der Rahmenbedingungen zur Eigenvorsorge mit gravierenden Folgen für die soziale Umverteilung und Wahrung der Solidarität begrenzt werden dürfen. Statt dessen warten die bislang für eine gehobene Niveausicherung in der Vorsorge entwickelten Grundsätze des (durch die Eigentumsgarantie und Art. 12 Abs. 1 GG gestützten) Vertrauensschutzes darauf, effektiv zu werden. Sie könnten im Zusammenhang mit dem Verfassungsprinzip Sicherheit unter Wahrung aller notwendigen Flexibilität des Vorsorgesystems die Kontinuität von Vorsorgeleistungen sichern bzw. einen „weichen“ Übergang auf abgesenkte Standards der Vorsorge über längere Zeit hinweg erzwingen. ${ }^{77}$ Der Gesetzgeber hat diesem Gedanken in Bezug auf die Verlängerung der Bezugszeiten von Hartz IV-Leistungen für ältere arbeitslose Arbeitnehmer Raum gegeben. Derartige und weitere gehaltvolle Maßgaben einer Niveausicherung des Sozialstaates erfließen nicht zuletzt dem Verfassungsprinzip der Sicherheit des Sozialstaates, wonach die Sicherheit, welche soziale Vorsorge ermöglicht, auch jenseits der schon durch das Rechtsstaatsprinzip verfestigten Ansprüche eingefordert werden darf.

75 Rüfner, in: Isensee/Kirchhof (Hrsg.), Handbuch des Staatsrechts (Fußn. 20), Rdnr. 83 m. w. Nachw.

76 BVerfGE 115, 25 (42 ff., 45).

77 Pitschas (Fußn. 2), VVDStRL Bd. 64 (2005), S. 136. 


\section{Die Gegenposition: Keine „, Verantwortungsteilung“ im Sozialstaat}

Die Diskussion über die Folgen der Modernisierung von Vorsorge ist unausweichlich. Sie führt jedoch nach hiesiger Ansicht auch deshalb und dort in die Irre, wo sie am Paradigma des sog. Gewährleistungsstaates orientiert wird. Dessen Grundannahmen stützen, wie bereits angedeutet wurde, den Wandel der staatlichen Vorsorge zur sozialen Selbstregulierung nur vermeintlich ab. Der theoretische Rahmen, den Staats- und Verwaltungsrecht unter Bezugnahme auf andere Referenzgebiete als das Sozialrecht derzeit für die Reichweite staatlichen Rückzugs aus der primären Hoheitsverantwortung anbieten können, ${ }^{78}$ lässt sich unter den Bedingungen einer Legitimität einfordernden Strukturentwicklung der privatisierenden Gestaltung sozialer Sicherung durch Sozial- und Gesundheitspolitik nicht erfolgreich ,überstülpen“.

Vor allem eine „Verantwortungsteilung“ als Begründungskonstrukt für den Rückzug aus der staatlichen Sozialverantwortung kennt das Grundgesetz nicht. Richtig ist zwar, dass die Rechtsprechung des BVerfG niemals eine Pflicht anerkannt hat, die erreichten sozialen Standards von Rechts wegen abzusichern. Den subsidiären Sozialstaat zu verwirklichen, bleibt hiernach ein „offener Prozess“, in dem das Maß der Vorsorge zwar gegen die Ungleichbehandlung einzelner Gruppen und gegen den Entzug erworbener Ansprüche prinzipiell abgesichert wird, der Veränderung von Sozialleistungen aber im Übrigen nur ein Mindestsicherungsniveau entgegengesetzt werden soll. ${ }^{79}$ Doch ist damit der verfassungs- und gemeinschaftsrechtliche Spielraum einer abgewogenen und niveausichernden Interpretation allgemeiner Rechtsgrundsätze des Sozialraums Europa, der Europäischen Grundrechte-Charta im Rahmen des Lissabon-Vertrags, von nationalen verfassungsrechtlichen Staatszielbestimmungen und Verfassungsprinzipien nicht erschöpft. „Verantwortungsteilige“ Interpretationen sollten das Augenmaß für die soziale Funktion dieser Maßgaben i. S. entsprechender Untermaßverbote und Schutzpflichten zur Sicherstellung sozialer Vorsorge sowie entsprechender Strukturverschaffungspflichten bewahren.

In besonderem Maße gilt die Absage an den „,nackten“ Gewährleistungsstaat für die Frage nach der Vorsorgefähigkeit und Vorsorgebedürftigkeit von Familien. ${ }^{80}$ Sie sind primäre Institutionen der Gesellschaft, die im Unterhaltsverband den Angehörigen Sicherheit zu bieten vermögen. Angesichts der Schutzdirektive des Art. 6 Abs. 1 GG muss deshalb im Zweifelsfall bei ihnen diagnostizierter insuffizienter Familienunterhalt in

78 Dazu mit w. Nachw. Voßkuhle, in: Hoffmann-Riem/Schmidt-Aßmann/Voßkuhle (Hrsg.), Grundlagen des Verwaltungsrechts (Fußn. 25), § 1 Rdnr. 49 ff.; zur Gegenposition Pitschas, Maßstäbe des Verwaltungshandelns, GVwR, Bd. II, 2008, § 42 Rdnr. 13 m. Anm. 44.

79 Dazu auch Rüfner, in: Isensee/Kirchhof (Hrsg.), Handbuch des Staatsrechts (Fußn. 20), Rdnr. 80 ff., 83.

80 Vgl. zusammenfassend Fuchs, Die Familie im Sozialrecht, in: Eichenhofer (Red.), Familie und Sozialleistungssysteme, SDSRV Bd. 57, 2008, S. 7 (19 ff.); Zacher, in: Isensee/Kirchhof (Hrsg.), Handbuch des Staatsrechts (Fußn. 1), Rdnr. 29 f. 
besonderer Weise durch ausgleichende Förderleistungen des Staates kompensiert werden. Dafür streitet überdies das aus Art. 3 GG in Verbindung mit dem Sozialstaatsprinzip abgeleitete und zudem auf Art. 2 Abs. 1 GG gestützte persönlichkeitsrechtliche Strukturkonzept für die Lebenslage „Kindheit/Jugend“. Vor allem diesem erfließt ein spezifischer Schutzauftrag der Verfassung für ,,intergenerationelle Gerechtigkeit“ . 81

Namentlich geht es bei dieser um die Frage, ob den Staat im Rahmen seiner herausgehobenen Schutzverantwortung für den Familienverband vor allem innerhalb der Gruppe der Kinder- und Jugendlichen eine besondere Förderungsverantwortung für „Kindergerechtigkeit“ trifft. Denn mit Blick auf Kindesvernachlässigung, steigende Kriminalität und den Drogenmissbrauch Jugendlicher, aber auch in Hinsicht auf jüngere Erfahrungen, wonach $15 \%$ bis $18 \%$ der Kinder und Jugendlichen in Deutschland in Armut aufwachsen, scheint gerade die generationenübergreifende soziale Gerechtigkeit nicht gewährleistet. Den Folgen dieser ungleich verteilten Lebenschancen vermag der sog. Gewährleistungsstaat ebenfalls nicht zu steuern. Es bedarf vielmehr eines aktiven staatlichen Schutzes dieses bislang kaum wahrgenommenen ,sozialen Kapitals“. Die Lebenschancen innerhalb der Gruppe der Kinder- und Jugendlichen, mehr noch: die einer Generation und darüber hinaus dürfen vor der Verfassung nicht ungleich verteilt bleiben.

\section{F. Struktur- und Funktionswandel des „,Vorsorgeverhältnisses “}

\section{Wandel der funktionalen Perspektive}

Das „Vorsorgeverhältnis“ als rechtliches Gehäuse der Beitrags- und Leistungsbeziehungen sozialer Kranken- und öffentlicher Alterssicherung scheint in einer gewissen Distanz zu den voraufgehend entfalteten Modernisierungsbedarfen und -dynamiken zu stehen. Doch täuscht dieser Eindruck. Als fortbestehendes öffentlich-rechtliches Institut, das eben auch in der sozialen Kranken- und Rentenversicherung seinen Platz findet, ist es dem Typus des Verwaltungs- bzw. Sozialrechtsverhältnisses zuzurechnen, das in der Literatur bereits zu einem „Pfeiler einer neuen Systembildung“ hypostasiert wurde ( $P$. Häberle). Dies mag hier dahinstehen. Jedenfalls bildet es über seine verfassungsrechtliche Dimension hinaus ein öffentlich-rechtliches (Dauer-)Schuldverhältnis, das in sich die Einflüsse der Modernisierung des Sozialversicherungsrechts und damit den Paradigmawandel in der Kranken- wie Alterssicherung sowie dessen Folgen aufzunehmen vermag. Denn das „Vorsorgeverhältnis“ bleibt in seiner Grundlegung ein Kind des for-

81 Steiner, Generationenfolge und Grundgesetz, NZS 2004, S. 505 (506 ff., 508 ff.). 
mellen Sozialstaatsprinzips; 82 es hat an dessen Prozesshaftigkeit und hoher Veränderungsgeschwindigkeit seinen Anteil. Im Zeichen wachsender privater Vorsorge treten dann neben das öffentlich-rechtliche Sozialrechtsverhältnis die weiteren privatrechtlichen Versorgungsverhältnisse ergänzend hinzu.

Im Hinblick auf diese Mehrdimensionalität entfaltet das öffentlich-rechtliche Vorsorgeverhältnis eine in ihren rechtlichen Folgen weitgehend unterschätzte strukturierende Funktion: In den als Rechtsverhältnis begriffenen Versorgungsbeziehungen sind die verschiedenen Einzelmomente der für die individuellen Versorgungs-, Anwartschaftsund Leistungsversprechen relevanten Lebenssachverhalte in ihren Tatsachengrundlagen und in ihrem verfassungs- und sozialversicherungsrechtlichen Kontext erfassbar und einander zuzuordnen. Die zugrundeliegenden Sachstrukturen, die wechselseitigen Bezüge zwischen einzelnen Freiheitseinschränkungen und Belastungen sowie Rechten, Handlungsmöglichkeiten aber auch Reaktionsnotwendigkeiten in Bezug auf Wandlungen des zugrundeliegenden Sicherungssystems treten dabei nicht nur deutlicher hervor, sondern sie werden personal dem jeweiligen Anspruchsinhaber zugeordnet. Oder, um dies mit Blick auf die in § 38-59 SGB I geregelten Grundsätze des Leistungsrechts auszudrücken - hier zeigt sich übrigens die Zwischenstellung des Sozialrechts zwischen öffentlichem und Privatrecht -: Für die im „Versorgungsverhältnis“ auf der Grundlage von Beitrag und Leistung festgezurrten Gegenseitigkeitsabreden bzw. -versprechen besteht eine clausula rebus sic stantibus mit der Konsequenz, dass sich das Netzwerk wechselseitiger Verknüpfung zwischen Versprechen und Erwartungen, Ansprüchen und Versorgungszusagen, Pflichten und Handlungsmöglichkeiten je nach Wandel der individuellen Situation verändern oder sogar gänzlich entfallen kann. Auch der Gedanke des Vertrauensschutzes nimmt in diesem Rahmen rechtsförmige Gestalt an; er wird individualisiert und flexibilisiert. 83

Diese Feststellung trifft zunächst und einerseits die im ,neuen“ Vorsorgerecht verankerte Umstellung vom Verwaltungsakt auf den Vertrag als künftig zentraler Handlungsform der Vorsorgevereinbarung und -leistung. Insoweit darf an die dargestellte Vertragskonzeption sozialstaatlicher Modernisierungspolitik im Rentenversicherungs- und Krankenversicherungsrecht erinnert werden. Der Rückbezug auf Verträge oder vertragsähnliche Gebilde als allgemeiner Grundsatz der Vorsorgemodernisierung führt wegen des veränderten Bedingungsgefüges zwischen Rechten, Pflichten und Handlungsmöglichkeiten unter den Vertragsparteien zu einem Funktionswandel des „Vorsorgerechtsverhältnisses“. Es erfasst nunmehr auch die Indienstnahme der Privatautonomie für Zwecke der Versorgung. Daraus können a la longue erhebliche Gefährdungen der

82 Dazu Zacher, in: Isensee/Kirchhof (Hrsg.), Handbuch des Staatsrechts (Fußn. 1), Rdnr. 82; Pitschas, Formelles Sozialstaatsprinzip, materielle Grundrechtsverwirklichung und Organisation sozialer Dienstleistungen, VSSR 1977, S. 141 ff.

83 Hase, Das Verwaltungsrechtsverhältnis, Überlegungen zu einem Grundbegriff des Öffentlichen Rechts, DV 38 (2005), S. 453 ff. 
individuellen Vorsorgepositionen auf Seiten der präsumtiven Vorsorgeempfänger resultieren.

\section{Strukturwandel des ,,Vorsorgeverhältnisses “ und staatliche Auffangverantwortung}

Seiner Struktur nach wandelt sich im Zuge dieser Entwicklung das ursprünglich einheitlich öffentlich-rechtliche Vorsorgeverhältnis zum „gemischten“ oder sogar privatrechtlichen Vertragsverhältnis. Die in dieser Transformation liegende Ausweitung der vertraglichen Konkretisierung von Rechtsnormen in der sozialen Vorsorge ${ }^{84}$ geht darüber hinaus einher mit einer gewissen Asymmetrie der Vertragsbeziehungen bei der Indienstnahme der Privatautonomie zu Vorsorgezwecken. Hierin liegt ein weiterer maßgeblicher Strukturaspekt im Wandel des „Vorsorgeverhältnisses“. Denn in den Beziehungen zu den Vertragspartnern fehlt es bei den privatrechtlichen Vorsorgebeziehungen von vorneherein an einer wirklichen Vertragsparität. Das Vertragskonzept sozialer Vorsorge vermag seine Funktion nämlich nur dann zu erfüllen und den Verlust der ehedem bzw. durch die Handlungsform des Verwaltungsakts gewährleisteten Rechtssicherheit zu kompensieren, wenn alle Vertragspartner ,,mit gleichlangen Spießen“ handeln würden. Das ist aber nicht der Fall; die betätigte Eigenverantwortung in der Vorsorge beruht nicht auf dem erforderlichen $\mathrm{Ma}$ an wirkmächtiger Vertragsautonomie. M. a. W. verändern sich Grund und Rahmen individueller Vorsorge, die im rechtsförmigen Sozialrechtsverhältnis geborgen sind. Zum Ausgleich dessen bedarf es im Gegenzug der staatlichen Auffangverantwortung gegenüber allfälligen Vorsorgerisiken.

\section{Staatliche Regulierung als Strukturkonsequenz}

Gewinnt auf diese Weise das „Vorsorgeverhältnis“ neue Funktionen und Strukturen als Privatrechtsverhältnis - daneben bleibt für die Grundsicherung und überlagernd das bisherige öffentlich-rechtliche Dauerschuldverhältnis bestehen -, so sind aus diesem Befund risikoreicher Netze von Vertragsbeziehungen Konsequenzen zu ziehen. Diese betreffen einerseits die unabweisbare Unterwerfung des gelenkten privatrechtlichen Vertragswettbewerbs unter staatlich-direktive Regulierung, die ihrerseits über unterschiedliche Ansatzpunkte verfügt. ${ }^{85}$

84 Fuchs/Preis, Sozialversicherungsrecht (Fußn. 18), § 48 I; In der Staatsrechtslehre wird von einer „Kontraktualisierung im Öffentlichen Recht“" gesprochen, s. Gromitsaris, Kontraktualisierung im Öffentlichen Recht, JöR N. F. Bd. 57 (2009), S. 255 ff.

85 Pitschas, Jenseits der Rentenreform 2001, Verfassungsdirektiven kapitalgedeckter privater Altersvorsorge im 21. Jahrhundert, VSSR 2002, S. 187 (201, 204 f.). 
So geht es zum einen um die Beaufsichtigung der Förderung privater Altersvorsorge im Rahmen steuerlicher Anreize für den Abschluss privatrechtlicher Vorsorgevereinbarungen. Auf der anderen Seite und insbesondere auf dem Feld der ergänzenden privaten Rentenversicherung hängen selbstverantwortete Investitionen in die Altersvorsorge von der künftigen Entwicklung der Kapitalmärkte und der Besteuerung der Geldanlagen ab. Privatanleger, die größere Geldbeträge in die private Rentenversicherung einzahlen, müssen im Gegenzug auf feste Renten mit langer Investitionsphase und dann langer Laufzeit vertrauen dürfen. Gleiches gilt im Übrigen für die Leistungskraft privater Krankenversicherung im Rahmen selektiver Verträge.

Die vorausschauende Regulierung muss insofern „intelligenten“ Aufsichtsinstanzen anvertraut werden. Bedauerlicherweise ist davon in den bedenkenswerten Vorschlägen des Gutachtens zum modernisierten Arbeits- und Sozialrecht für eine alternde Gesellschaft auf dem 67. DJT 2008 nicht die Rede, wenn und soweit gefordert wird, dass „während der Erwerbsphase ... verstärkt in die private Altersvorsorge investiert werden (muss)“ “. ${ }^{86}$ Plädiert wird dort für eine steuerfinanzierte Sockelrente, ohne allerdings der dem jetzigen Rechtszustand immanenten Sicherung zukünftiger Vorsorgeansprüche die geschuldete Aufmerksamkeit zu widmen.

Verfassungsrechtlich fragwürdig ist darüber hinaus die derzeit im SGB II verankerte Grundsicherung für Arbeitsuchende, soweit sich diese des Vertragskonzepts im Rahmen des „Vorsorgeverhältnisses“ bedient. Nach $§ 15$ Abs. 1 SGB II soll nämlich in der mit jedem erwerbsfähigen Hilfebedürftigen abzuschließenden Eingliederungsvereinbarung eine den Hilfebedürftigen ,aktiv“ in den Eingliederungsprozess einbindende Stoßrichtung des Verwaltungshandelns zum Ausdruck kommen. Soweit so gut! Doch enthält die Einbindung, die auch rechtstatsächlich in der sozialen Wirklichkeit Platz greift, sowohl fördernde als auch fordernde Momente; der öffentlich-rechtliche Vertrag entfaltet selbstbelastende Konsequenzen für den erwerbsfähigen Hilfebedürftigen. Es handelt es sich m. a. W. doch um eine mittelbar wirkende „Zwangsvereinbarung“, die des Näheren eine Vielzahl rechtlicher Fragen aufwirft. ${ }^{87}$

Geht man ihnen nach, so steht die aufgrund der gesetzlichen Druck- und Sanktionsmechanismen schwache Verhandlungsposition des Arbeitssuchenden einer Qualifikation als „Vertrag“ statt einer ,gemischten Handlungsform“ des öffentlichen Rechts entgegen. $\mathrm{Zu}$ fragen ist überdies, ob die gesetzliche Ausgestaltung der Eingliederungsvereinbarung noch verfassungskonform ist. Art 12 Abs. 2 GG entfaltet nicht zuletzt eine objektiv-rechtliche Fernwirkung, die nach dem Willen des Verfassunggebers auch gegen mittelbaren Zwang zur Arbeitsaufnahme unterhalb des Existenzsicherungsgebots schützt.

86 Preis (Fußn. 31), S. B 68 f., B 109, B 115 f.

87 Vgl. auch Ebsen, in: v. Wulffen/Krasney (Hrsg.), FS 50 Jahre BSG (Fußn. 53), S. 737. 


\section{Organisationsrechtliche Konsequenzen}

Unter der Einwirkung des Verfassungsprinzips Sicherheit darf sich jedenfalls der Staat seiner Aufsichtsaufgabe nicht entziehen. Privatrechtlich vereinbarte Kapitaldeckung der Vorsorgeverträge ist ein gefährliches Unterfangen, wie nicht nur die Nachkriegszeit in Deutschland, sondern auch internationale Erfahrungen lehren. Darauf wurde bereits hingewiesen. Erkennt man deshalb die Unverzichtbarkeit gleichzeitiger staatlicher Vorsorgeregulierung an, die sich insgesamt in das Regulierungsrecht für Finanzdienstleistungen und Kapitalmärkte einerseits, für Daseinsvorsorgeleistungen andererseits einfügt, ${ }^{88}$ dann stellt sich die Frage nach der adäquaten Organisation - der selbstredend auch eine (verfassungs-)rechtliche Dimension innewohnt. Sie hat darüber hinaus gemeinschaftsrechtliche Konsequenzen im Rahmen der von der Kommission in Aussicht gestellten „Modernisierung der Systeme sozialen Schutzes“. 89

Als Folge dessen werden national als staatliche Aufsichtsinstanzen hinsichtlich der sog. Riester- bzw. Rürup-Rente die Deutsche Rentenversicherung/Bund und das Bundesversicherungsamt tätig. Hinsichtlich der finanzpolitischen Fragestellungen ist die Bundesanstalt für Finanzdienstleistungsaufsicht zuständig. Gegenüber allen diesen Institutionen besteht jedoch im Zeichen der Finanzmarktkrise der Verdacht der Ineffektivität und Effizienzdefizite. Die Empfehlung muss daher lauten, eine selbständige Regulierungsbehörde für die soziale Vorsorge auszuformen. Organisationsentwicklung lautet sonach der Auftrag des formellen Sozialstaatsprinzips. Er wird von der europarechtlichen Frage nach einer institutionellen Regulierung durch die Gemeinschaft überlagert.

\section{G. Soziale Vorsorge im ausländischen und vergleichenden Kontext}

\section{Zwischenbilanz und Notwendigkeit der vergleichenden Perspektive}

Ziehen wir eine Zwischenbilanz. Der Bedeutungswandel der sozialen Vorsorge ist nach alledem unverkennbar. Die ursprünglich als ergänzend im geringen Umfang angesehene private Altersicherung geht in eine partielle Abkehr vom Umlageverfahren über. Der Wechsel zur Kapitaldeckung von Anwartschaften auf Altersrente sowie zur privatisierten Altersvorsorge wird in einem Ausmaß angestrebt, das nur noch Raum für eine staatliche Grundsicherung belassen möchte.

88 Wesentlich ist dabei das Zertifizierungsverfahren, s. dazu Fuchs/Preis, Sozialversicherungsrecht (Fußn. 18), § 48 I 3 c (S. 821 f.), für das grds. die Bundesanstalt für Finanzdienstleistungsaufsicht zuständig ist.

89 Commission of the European Communities, Renewed social agenda: Opportunities, access and solidarity in the 21st century Europe, 2.7.2008, COM (2008), 412 final. 
Vor einem sukzessiven Systemwechsel steht, wie ausgeführt, auch die gesetzliche Krankenversicherung. Dagegen erhielt die Pflegeversicherung von Anfang an eine „offene" Grundausstattung. Im Mittelpunkt der Systemveränderung in der Gesundheitsversorgung steht vor allem der Übergang zu einer allgemeinen Basiskrankenversicherung mit zentraler Finanzierung durch einen staatlichen Gesundheitsfonds. Sie unterliegt dem Wettbewerb unter den gesetzlichen Kassen und dieser mit der privaten Krankenversicherung bei fortbestehender Trennung beider, steuert aber dessen Ansatz und Verlauf über einen regulierten Vertragswettbewerb und entsprechende Eingriffe in den Arzneimittelmarkt. Der Versicherte und künftige Patient wird als informierter Partner auf dem Gesundheitsmarkt und als Gesundheits- bzw. Rentenbürger mit Pflichten zur Selbstregulierung in diese Steuerung einbezogen. Es bestehen Möglichkeiten einer ergänzenden Wahlversorgung im Gesundheitswesen mit neuen Versorgungsarrangements des Sozialstaats.

\section{Unbehelflichkeit der nationalen Diskussion über die Entstaatlichung sozialer} Vorsorgeaufgaben

Für die Analyse und Deutung dieser Entwicklung ist das staats- und verwaltungsrechtliche Theorem des sog. Gewährleistungsstaates nicht anschlussfähig.

Die ihm fälschlich unterlegte „Verantwortungsteilung“ zwischen Staat und Markt besteht nicht und die Ausführungen hierzu erklären auch nichts; sie tragen nicht einmal als empirische Beobachtung. Denn der prozesshafte Sozialstaat „managt“ nach wie vor die ihm vom Grundgesetz ungeteilt zugewiesene Verantwortung für Vorsorge als eine „Regierung des Sozialen“. Oder wie die einschlägigen Überlegungen hierzu in der Literatur formulieren: „,.. der Übergang zu einer sozialstaatlichen Steuerungslogik der gesellschaftlichen Selbstaktivierung - und die damit verbundene Wiederentdeckung des Sozialen im Individuum - lässt sich ... gewinnbringend als Ausdruck einer neuen politischen Rationalität, als Wandel in der Programmatik und den Techniken gesellschaftlicher ,Regierung' beschreiben und begreifen". 90 Diesem zutreffenden Denkansatz gegenüber erscheinen auch governancebezogene Überlegungen als blasse Deduktion verzerrt wahrgenommener Wirklichkeit, selbst wenn sie international vergleichend ansetzen.

\section{Entwicklung sozialer Vorsorge in vergleichender Perspektive}

Freilich haben der Verwaltungs- und Rechtsvergleich ihre Tücke. „Verwaltungssysteme“ des einen Staates lassen nicht völlig die „Spiegelung“ zu denen eines anderen Staates zu; das gewonnene Bild ist in der Regel verzerrt. Gleiches gilt für den Rechts-

90 Blömeke, Die Regulierung nicht-staatlicher Alterssicherung, Deutschland und Großbritannien im Vergleich unter Berücksichtigung von Staatstheorie, Verfassungs- und Europarecht, Berlin 2007, S. $13 \mathrm{ff}$. 
vergleich. Er ist zwar für das Sozialrecht unverzichtbar. Doch mögen einige knapp skizzierte Beispiele für den Bereich der Vorsorge die Schwierigkeiten des Sozialrechtsvergleichs beleuchten. ${ }^{91}$

Schon der Begriff der ,,sozialen Sicherheit" kann in Untiefen führen. Vermeintlich klar umrissen, ist er erst jüngeren Datums. Er stellt die sprachliche Übersetzung von „Social Security“, also der Leitidee der Sozialgesetzgebung des „,new deal“ unter Franklin D. Roosevelt aus dem Jahre 1935 dar. Das Konzept wurde weltweit schnell übernommen; es bezeichnet einen komplexen Zusammenhang von Phänomenen und Lösungsmustern sozialer Sicherung. Dass es aber in seinem Ursprungsland, den Vereinigten Staaten von Amerika, allgemein sprachlich häufig mit Alterssicherung gleichgesetzt wird, erklärt sich aus der Tatsache, dass ein großer weiterer Sicherungskomplex, nämlich die Gesundheitsversorgung, dort teils der Eigenverantwortung des Einzelnen überlassen bleibt, teils in öffentlicher Gestalt anders erfasst wird. Dagegen wird in Deutschland die Krankenversicherung in ihrer über ein Jahrhundert alten Geschichte seit Bismarck, ausgestaltet als öffentliche Zwangsversicherung für die meisten Bevölkerungsgruppen und im Übergang zur Basiskrankenversicherung befindlich, wie „,selbstverständlich“ mitgedacht. Das Beispiel zeigt bereits, dass auch zwischen wirtschaftlich „vergleichbaren“ Nationen sehr unterschiedliche Wahrnehmungsfelder sozialer Vorsorge bestehen können, welche sich bei unzureichender Reflexion in wissenschaftlichen Analysen als Fehleinschätzungen fortsetzen können. ${ }^{92}$

Es darf also nicht unbeachtet bleiben, dass in Staatengruppen mit gleichen oder ähnlichen „Leitideen“ erhebliche Unterschiede in den gewählten Absicherungsformen und mechanismen bestehen können. Um so mehr gilt dies natürlich für Staaten mit prinzipiell verschiedenen Gesellschaftsentwürfen, wie sich etwa am Beispiel der Vorsorge für behinderte Menschen in Europa und Asien zeigt. ${ }^{93}$ Dennoch soll im Folgenden nicht auf einige vergleichende Bemerkungen verzichtet werden. Sie ergehen zunächst mit Blick auf die Sicherung der nachhaltigen Wirksamkeit sozialer Vorsorge durch Rentenund Krankenversicherung als künftige Aufgaben des Sozialschutzes in der EU. Den erweiterten Perspektiverahmen bilden sodann die Staaten der OECD.

Die in den Feststellungen hierzu eingebetteten Erkenntnisse zur Entwicklung sozialer Vorsorge formulieren für den „lernenden Staat“, also für den prozesshaft verankerten Sozialstaat Deutschland eine unablässige Herausforderung. Doch hat das Grundgesetz diese von Anfang an gleichsam vorsorglich in seiner Zuwendung zur internationalen Rechtsordnung und mit dem Prozesscharakter des Sozialstaats angenommen: Es be-

91 Pitschas, Die Bedeutung von Modellen für den Transformationsprozess, in: v. Maydell/Nußberger (Hrsg.), Transformation von Systemen sozialer Sicherheit in Mittel- und Osteuropa, Berlin 2000, S. 323 (324 ff.).

92 Pitschas/Iff, Sozialrechtsinfrastruktur als Entwicklungsbedarf, in: dies. (Hrsg.), Soziale Sicherung in Brasilien und Peru, Speyerer Forschungsberichte Bd. 102, 1991, S. 1 (4 ff.).

93 Vgl. etwa Leisering, Sozialpolitische und -rechtliche Gestaltung der Behindertenpolitik in Asien, in: v. Maydell/Pitschas/Schulte (Hrsg.), Behinderung in Asien und Europa im Politik- und Rechtsvergleich, Baden-Baden 2003, S. 426 (428 ff., 433). 
greift ihn ebenfalls als eine nachhaltig wirksame staatliche Verpflichtung, aber nicht im Sinne einer Bewahrung des bestehenden, sondern als ständige Aufforderung an die Bewältigung neuer sozialer Herausforderungen. Das Sozialstaatsprinzip sichert auf diese Weise nicht den Status Quo, sondern es will in Abstimmung mit dem Verfassungsprinzip Sicherheit die Überwindung befördern helfen. Oder m. a. W.: Das Sozialrechtsverhältnis wird entterritorialisiert. Nur so können Sozialstaat und soziale Vorsorge auf Dauer wirklich gesichert werden. ${ }^{94}$

\section{Ausländische und supranationale Leitbilder für Vorsorgemodernisierung}

\section{Die Niederlande als Leitbild deutscher Modernisierungsanstrengungen}

Es überrascht nicht, dass aus vergleichender Perspektive auch in anderen Staaten Europas und insbesondere in der EU die soziale Sicherheit - und mit ihr der Sektor der sozialen Versorgung - mehr oder weniger dem gleichen staatlichen Strategiewandel und entsprechenden gesellschaftlichen Transformationsprozessen unterliegt. Zunehmend werden die europäischen Gesellschaften auf dem Weg in den „Europäischen Sozialstaat" zum Subjekt ihrer eigenen und kollektiven Sicherheitsbemühungen erkoren.

In der diesen Wandel abbildenden Versicherungsgestalt sozialer Vorsorge - z.B. der Einrichtung von Altersvorsorge durch Betriebsrenten oder auch den Abschluss von Lebensversicherungen - sieht sich die Altersvorsorge zugleich und selbst neuartigen Sicherheitsbedrohungen wie der Besteuerung der Altersrenten oder auch der Verlegung der Altersgrenze für die Regelaltersrente von bisher 65 Jahren auf das 67. Lebensjahr ausgesetzt. Dies geschieht stufenweise wie in Deutschland, letztlich aber ohne Ausnahme für alle oder aber auf der Grundlage freiwilliger Entscheidungen wie in den Niederlanden, wohlbemerkt dort ohne Abschläge für frühere Inanspruchnahme der Renten.

Überhaupt nehmen die Niederlande in den bisherigen Bemühungen um eine in der EU abgestimmte Politik sozialen Schutzes eine herausragende Rolle ein. ${ }^{95}$ In der $\mathrm{Ar}$ beitsförderung fallen die niederländischen Bemühungen auf, Sozialleistungen in den Dienst der Eingliederung betroffener arbeitsloser Bürger in den ersten Arbeitsmarkt zu stellen; nicht von ungefähr geht die Rede davon, die Modernisierung der deutschen Bundesanstalt für Arbeit zu einer „Bundesagentur“ sowie der damit verbundene Einsatz flexibler Vermittlungsinstrumente oder auch die Erfindung der deutschen Personal-Service-Agentur sei dem niederländischen Muster einer institutionellen Re-Formulierung

94 Kingreen, Die Universalisierung sozialer Rechte im europäischen Gemeinschaftsrecht, in: Giegerich/Zimmermann (Hrsg.), Wirtschaftliche, soziale und kulturelle Rechte im globalen Zeitalter, Berlin 2008, S. 109 (116).

95 Allgemein dazu in seinem Beitrag Eichenhofer, Gleiche Ziele, vergleichbare Wege der Gesundheitspolitik in den Niederlanden und Deutschland?, in: Pitschas (Hrsg.), Die Gesundheitsreform 2007 als Herausforderung an Beruf und Status der Vertragszahnärzte, 2009, S. 207 ff. (i. Ersch.). 
der Beschäftigungspolitik gefolgt. In der Alterssicherung verbinden die Niederlande eine Grundrente für alle Bewohner mit der ausgebauten betrieblichen Altersvorsorge. Wer will, der mag - weniger stringent reguliert als in der Schweiz - noch die eigenfinanzierte Lebensversicherung ,draufsatteln“.

Schließlich folgt auch die künftige Gesundheitsvorsorge in Deutschland dem niederländischem Beispiel: Sie verbindet die öffentliche und private Krankenversicherung in einer sog. Bürgerversicherung auf der Grundlage einer Einheitsprämie, die wir hierzulande als „Kopfpauschale“ bezeichnen. Selbstverständlich bleibt die Frage zu beantworten, die jeder staatlichen Sozialpolitik gestellt wird, ob nämlich die Synthese von Freiheit und sozialem Ausgleich erreicht wird. Nicht auszuschließen ist aber, dass die hiesige Debatte um die richtige Organisationsform der Gesundheitsversorgung im Angesicht von demografischem Wandel und medizinischem Fortschritt durch das niederländische Beispiel weiterhin angestoßen wird.

\section{Entwicklungslinien sozialer Sicherheit im Recht der Europäischen Union}

Dessen bedarf es angesichts der bereits skizzierten Probleme in der sozialen Krankenversicherung Deutschlands mit dem europäischen Vergabe- und Wettbewerbsrecht unbedingt. Erforderlich wäre z.B. eine einheitliche Wettbewerbsordnung der Krankenversicherung, deren Errichtung nach der hier vertretenen Auffassung mit Überlegungen zu verbinden wäre, wie der Gegensatz zwischen sozialer und privater Krankenversicherung aufzuheben wäre. Irrtümlicherweise wird hiergegen stets mit dem „Kampfbegriff“ der ,Einheitsversicherung“ argumentiert. 96

Eine „Hilfestellung“ auf dem zukünftigen Weg versucht die Europäische Union (EU) zu geben. Diese ist sich freilich dessen bewusst, dass die Hauptverantwortung für die Gesundheitsversorgung in den Mitgliedstaaten der Gemeinschaft liegt. In der Tat hat die EU auf dem Gebiet der sozialen Sicherheit nur geringe Kompetenzen. Das Gemeinschaftsrecht belässt die Systeme der sozialen Sicherung in den Mitgliedstaaten, insbesondere deren Sozialversicherung, weitgehend in nationaler Verantwortung. Der soziale Vorsorgestaat ist ein „nationalstaatliches Projekt“".

Allerdings sollte spätestens seit dem Lissabonner Vertrag97 der Schwerpunkt der Bemühungen der EU nicht mehr nur in der Koordination sozialrechtlicher Regelungen für Fälle mit Auslandsbezug innerhalb der Gemeinschaft gesehen werden. Die Weichen sind vielmehr in Richtung Harmonisierung gestellt. Dabei geht es nicht nur um die in den Verordnungen Nr. 1408/71 und 574/72 erlassenen notwendigen Kollisionsregeln. Rückwirkungen auf die soziale Vorsorge i. S. des Ausgleichs von Unterschieden in Vorsorgebedarfen und -fähigkeit haben auch die Antidiskriminierungsregeln der Ge-

96 Dazu näher noch Pitschas, Gesundheitsstrukturreform - Einheitsversicherung oder Trägervielfalt?, in: Bitburger Gespräche, Jahrbuch 1996, München 1996, S. 15 (23 ff.).

97 In seiner Gestalt als Vertrag über die Europäische Union vom 13.12.2007, ABl. Nr. C 306/1 vom 17.12.2007, vgl. a. a. O. (Fußn. 7). 
meinschaft. Hierzu wird es demnächst den Vorschlag der Europäischen Kommission für eine weitere Antidiskriminierungsrichtlinie geben, die z.B. zukünftig auch behinderte Menschen erfassen will. Der neue Entwurf ist ferner bemüht, Diskriminierung insbesondere aus Altersgründen zu verbieten. Und nicht zuletzt hat bereits die in der Beschäftigungspolitik verwirklichte „Methode der offenen Koordination“ auch in der Gesundheitsversorgung ihren Weg in das Gemeinschaftsrecht gefunden. ${ }^{98}$

Darüber noch hinausgehend hat die Kommission eine neue sozialpolitische Agenda vorgeschlagen. Dazu hat sie eine vorbereitende Mitteilung mit dem Titel „Chancen, Zugangsmöglichkeiten und Solidarität: eine neue gesellschaftliche Vision für das Europa des 21. Jahrhunderts" veröffentlicht. ${ }^{99}$ In dieser verweist sie auf den bestehenden „soliden Rechtsrahmen“ der Gemeinschaft zur Sozialpolitik. Ihn gelte es unter Berücksichtigung der sich verändernden „sozialen Realitäten“, aber auch des EUReformvertrags zu überprüfen sowie ggf. anzupassen.

\section{Ein neuer Gesellschaftsvertrag für soziale Vorsorge und Sicherheit aus}

\section{internationaler Perspektive?}

Die Sicherung der nachhaltigen Wirksamkeit sozialer Vorsorge verkörpert nicht nur eine künftige Aufgabe des Sozialschutzes in der EU. Sie steht ebenso im Zentrum internationaler Sozialpolitik. Denn die sich wandelnden Rahmenbedingungen für soziale Vorsorge verändern sich weltweit; insofern gibt es übereinstimmenden Handlungsbedarf: In allen OECD-Staaten sind politische Entscheidungsträger immer weniger bereit, Lohnnebenkosten bzw. Sozialversicherungsbeiträge anzuheben, um Belastungen der eigenen Wirtschaft zu vermeiden. Darüber hinaus sind es neben der demografischen Alterung mit den damit einhergehenden Rentenausgaben vor allem die Gesundheitskosten, die stärker steigen als die Inflation. Auch die Finanzierung anderer notwendiger Leistungen im Rahmen des Sozialschutzes ist ernsthaft gefährdet. Deutlich wird, dass ein neuer Konsens über Vorsorge angestrebt, aber auch international erst noch gefunden werden muss. 100

Lösungen im Einzelnen dürften sich dabei je nach Land aufgrund seiner Geschichte, Kultur und wirtschaftlichen Situation stark voneinander unterscheiden. So ist beispielsweise weithin eine sich verändernde Einstellung zur individuellen Verantwortung für die Alterssicherung festzustellen, doch variiert das Ausmaß der Risikoverlagerung vom Staat weg und hin zu den Arbeitgebern von Staat zu Staat. Gleichwohl lassen sich An-

98 Vgl. die erneuerte Sozialagenda (Fußn. 89), 412 endg. Punkt 5.3; Art. 5 Entwurf „PatientenRichtlinie“ (Fußn. 27): „Leitlinien“; s. ferner Becker, Der nationale Sozialstaat in der Europäischen Union: von Einwirkungen und Verschränkungen, in: Bĕlina/Kalsenská (Hrsg.), Pocta Petru Trösterovi k 70. narozeninám (Festschrift für Petr Tröster zum 70. Geburtstag), Prag 2009, S. 49 (60f.).

99 A. a. O. (Fußn. 89).

100 Hoskins, Soziale Sicherheit: Brauchen wir einen neuen Gesellschaftsvertrag?, IRSS Bd. 60 (2007), S. $163 \mathrm{ff}$. 
zeichen für einen dauerhaften Übergang zur privaten Risikoversicherung weithin beobachten. ${ }^{101}$

Die Diskussion über das für den Einzelnen insoweit noch zumutbare Risiko und über zu belassene Freiheitsspielräume berührt den Kern der ursprünglichen Sozialversicherungspolitik. Es erstaunt deshalb nicht, dass zahlreiche Fragen aufgeworfen werden, so etwa jene, wie die Bürger reagieren werden, wenn ihnen gleichzeitig verschiedene Angebote für die Alterssicherung sowie für die Kranken-, Invaliditäts- und Langzeitpflegeversicherung unterbreitet werden. Und was wird geschehen, so fragt man sich, wenn Regierungen die Bürger verpflichten, private Renten- und Krankenversicherungen abzuschließen, dazu jedoch fehlerhafte Entscheidungen getroffen werden, weil z. B. in der Altersvorsorge die Rendite zu gering ist oder die in Anspruch genommenen Versicherungsunternehmen, z. B. Pensionsfonds, ihre Insolvenz anmelden? 102

Die Folgen solcher Entscheidungen abfedernd, ist daher vielen Staaten der Übergang zu einem Mehrsäulensystem des vorsorgenden Sozialschutzes gemeinsam - verbunden mit öffentlichen Subventionsprogrammen zum Schutz armutsnaher Bevölkerungsgruppen. Indes darf die Entscheidung für eine solche gegliederte Altersvorsorge wiederum nicht zu größeren Einkommensunterschieden und zu einem Wachstum der von Armut betroffenen Bevölkerungsgruppen führen. Dies würde bedeuten, die bereits für die Sozialversicherungssysteme gerügte soziale Ungleichheit zu vertiefen. Interessanterweise ist das aber in jenen Staaten oft der Fall, die beschlossen haben, statt der ersten (öffentlichen) Säule des staatlichen Versicherungsschutzes den vom Privatsektor angebotenen Schutz der zweiten und ggf. den einer dritten freiwilligen Säule zu fördern.

Immer wieder stellt sich überdies das Problem, wie die weitere Beteiligung der Arbeitgeber an der Finanzierung der sozialen Vorsorge sichergestellt werden kann. Die Ausführungen des Gutachters für den 67. Deutschen Juristentag mit Blick auf die Situation Deutschlands lassen dabei wenig Problemtiefe erkennen, wenn sie nicht mehr vorschlagen als einen Ausbau der finanziellen Alterssicherung auf „vier Säulen“. Leitend sollte für eine Empfehlung eigentlich und vielmehr die Überlegung sein, dass sich der Produktionsfaktor „Arbeit“ nicht zu einem Hemmschuh entwickelt, wenn die Bundesrepublik Deutschland ihr bisheriges Wohlstandsniveau nicht verlieren will. Die Sicht menschlicher Bedingungen für Erwerbsarbeit darf aber nicht aus dem Blick geraten.

\section{Notwendigkeit eines dynamischen Rahmenkonzepts zur Förderung eigenverantwortlicher sozialer Vorsorge}

Die Palette möglicher Handlungsoptionen ist somit aus internationaler Perspektive breit aufgefächert. Der Zugriff auf eine bestimmte unter ihnen hängt einerseits von den

101 McKinnon, Für eine dynamische soziale Sicherheit: Ein Konzept für den Wandel und die Ausdehnung der Deckung, IRSS Bd. 60 (2007), S. 171 ff.; Hoskins (Fußn. 100), IRSS Bd. 60 (2007), S. 165.

102 Hoskins (Fußn. 100), IRSS Bd. 60 (2007), S. 165. 
Sicherungsbedarfen in dem jeweiligen Reformstaat ab. Dabei ist in der EU allgemein die alarmierende soziale Situation in einzelnen Mitgliedstaaten zu bedenken. Festzustellen ist die Transformation der Arbeitswelt mit ,aufbrechenden Lohnstrukturen“ namentlich in den europäischen Staaten. Prekarität und soziale Ausgrenzung, instabile Familien und zahlreiche andere empirisch wahrnehmbare Aspekte kennzeichnen die soziale Situation des Individuums bzw. seines Unterhaltsverbands, welche die Sicherheit im Rahmen seiner Vorsorge in Frage stellen. Das Ziel der Sicherheit des Individuums als ein zentrales Element der Vorsorge muss diesbezüglich unstreitig gestellt werden. Um zu klären, wie der künftige Sozialschutz in der EU zwischen Solidarität und Eigenverantwortung aussieht, bedarf es daher aus bisheriger Sicht eines neuen Gesellschaftsvertrags. Er hat die Vorsorgeverantwortung der Arbeitgeber, die das BVerfG bereits für den Arbeitsmarkt und die Arbeitsförderung bejaht hat, auch für den zukünftigen Sozialschutz verbindlich auszugestalten.

Hierfür wird ein Rahmenkonzept der dynamischen sozialen Sicherheit erforderlich, das nicht zuletzt die institutionelle Leistungsfähigkeit im Vorsorgesektor und dort auch die speziellen Vorsorgebedarfe bestimmter Bevölkerungsgruppen erfasst. So gibt es in einer Vielzahl von Staaten - ganz ähnlich wie in Deutschland - den Druck zu einer weiteren und schnelleren Anpassung der Institutionen sozialer Sicherheit. ${ }^{103}$ Hierauf bezogen ist inzwischen deutlich geworden, dass es nicht reicht, sich für eine Erweiterung der gesetzlichen Grundlagen einzusetzen. Dies gilt insbesondere für beitragsbezogene Systeme, deren Beitragsniveau unrealistisch hoch angesetzt worden ist. Zu erwähnen sind ferner das Problem der Verbesserung des Zugangs zur sozialen Sicherung und auch die Frage, ob die erbrachten Leistungen den von der Bevölkerung empfundenen Bedürfnissen tatsächlich entsprechen. Vernachlässigt wird schließlich, dass viele Träger der sozialen Sicherheit Unterstützung bei der Anpassung der Arbeitsmethoden in ihrer Organisation brauchen. Hierbei tritt die Notwendigkeit einer gesteigerten Kosteneffizienz neben die Frage, wie die Leistungsfähigkeit der Organisation durch die Nutzung der Informations- und Kommunikationstechnologie verbessert werden könnte. Ebenso wäre aufzugeben, durch sog. benchmarking bessere Ergebnisse in der Leistungsperformance zu erreichen.

Allerdings sollte nicht vergessen werden, dass hinter diesen „Modernisierungsansätzen“ grundsätzliche Fragen einer menschenwürdigen Vorsorgepolitik aufscheinen. So bleibt z.B. die „Gretchenfrage“ der Kontingentierung von Krankenversicherungsleistungen unter ökonomischen Aspekten: Wollen wir Patientenautonomie in Zukunft oder Leistungsrationierung? Es könnte sehr wohl sein, dass Vorsorge- wie Sozialpolitik insgesamt künftig als Teil sowohl des „Vorsorgeproblems“ als auch seiner Lösung verstanden werden müssen.

103 Vgl. z.B. Turner, Das Mindestrentenalter in der Sozialversicherung von OECD-Ländern: 1949 2035, IRSS Bd. 60 (2007), S. 89 ff. 


\title{
Soziale Vorsorge als Funktion
}

\author{
Ingwer Ebsen
}

I. Einleitung: Funktionale Systematisierung des Sozialrechts

II. Soziale Vorsorge 109

1. Soziale Vorsorge als Daseinsvorsorge 109

2. Soziale Vorsorge als Sozialversicherung 112

a) Vorsorge gegen soziale Risiken 114

b) Beitragsfinanzierung sozialer Vorsorge $\quad 115$

c) Sozialer Ausgleich 118

d) Versicherungszwang 121

3. Soziale Vorsorge über Sozialversicherung hinaus 122

a) Der partielle Rückzug der gesetzlichen Rentenversicherung
zugunsten staatlich geförderter privater Altersvorsorge

b) Ansätze zu sozialer Vorsorge mit Pflichtcharakter und sozialem $\begin{array}{ll}\text { Ausgleich in Privatversicherungsform } & 126\end{array}$

III. Vorsorgeverhältnis als Rechtsbegriff 129

\section{Einleitung: Funktionale Systematisierung des Sozialrechts}

Wer sich mit dem Vorsorgeverhältnis beschäftigt, kommt nicht um eine intensive Befassung mit dem Werk von Hans F. Zacher herum. Seine an Funktionen sozialer Sicherung ansetzenden Kategorisierungen und Begrifflichkeiten sind prägend geworden für das Verständnis des inneren Systems des deutschen Sozialrechts. In der Auseinandersetzung mit der überkommenen Dreiteilung des nationalen Sozialrechts in soziale Fürsorge, Versorgung und Sozialversicherung hat Zacher seine „Anatomie des Sozialrechts" 1 entwickelt, in welcher sich die soziale Vorsorge - selbstverständlich im Sozialrecht das Feld der Sozialversicherung, aber funktional eben etwa auch die Beamtenversorgung und die spezifischen Alterssicherungssysteme der „Freien Berufe” - von der sozialen Entschädigung und der nur bedingt intern unterscheidbaren sozialen Hilfe und Förderung abgrenzen lässt. Dieses System hat Zacher seiner „Einführung in das Sozial-

1 Zacher, Zur Anatomie des Sozialrechts, SGb. 1982, S. 329 ff. 